\title{
Management of the aphid, Myzus persicae (Sulzer) and the whitefly, Bemisia tabaci (Gennadius), using biorational on capsicum under protected cultivation in India
}

Harshdeep Singh ${ }^{1}$ and Neelam Joshi ${ }^{*}$

\begin{abstract}
The peach aphid, Myzus persicae (Hemiptera: Aphididae) and silver leaf whitefly, Bemisia tabaci (Hemiptera: Aleyrodidae) are the major pests of capsicum under protected cultivation. The entomopathogenic fungi (EPF) are environmentally safe than the chemical pesticides. In the present study, different EPF formulations, Beauveria bassiana Balsamo (Vuillemin), Lecanicillium lecanii (Zimmerman) Viegas, Metarhizium anisopliae (Metschnikoff) Sorokin, were evaluated along with Azadirachtin 1\% for the management of the aphid and the whitefly on capsicum under protected conditions. Result showed that talc formulation of L. lecanii MTCC 956 at 10 and $12 \mathrm{~g} / \mathrm{l}$ caused 60.5 and $61.6 \%$ population reduction for aphid and 60.0 and $61.6 \%$ population reduction for whitefly, whereas Azadirachtin $1 \%$ at 4 and $5 \mathrm{ml} / \mathrm{l}$ caused 71.2 and $74.7 \%$ population reduction for aphid and 68.5 and $71.0 \%$ population reduction for whitefly after 3rd spray, respectively and were effective in reducing aphid and whitefly populations on capsicum recommending its organic production under protected cultivation and could be a part of integrated pest management program.
\end{abstract}

Keywords: Capsicum, Entomopathogenic fungi, Myzus persicae, Bemisia tabaci, Protected cultivation

\section{Background}

Capsicum is an annual herbaceous vegetable crop also known as sweet pepper and is broadly cultivated in India and all over the world as table food for its exquisite taste and good flavor. The active chemical component of sweet pepper called "capsaicin" has antioxidant, anti-carcinogenic, and anti-diabetic properties (Gupta et al. 2016). Temperature, relative humidity, and energy may influence the growth of sweet pepper under open field cultivation. Under protected cultivation, all these factors are maintained for its efficient productivity.
The aphids, Myzus persicae (Sulzer) and Aphis gossypii (Glover); whitefly, Bemisia tabaci (Gennadius); mite, Polyphagotarsonemus lactus (Banks); and thrips, Scirthorips dorsalis (Hood) are economic pests which infest crops under protected cultivation. They are sucking pests, which effect the growth and hence lead to reduction in crop yield. Among these, M. persicae (Hemiptera: Aphididae) and B. tabaci (Hemiptera: Aleyrodidae) are major insect pests, which cause vector borne viral diseases and their damage includes chlorosis, necrosis, wilting, stunting, flower and fruit abortion, leaf distortion, and

\footnotetext{
* Correspondence: neelamjoshi_01@pau.edu

${ }^{2}$ Department of Entomology, Punjab Agricultural University, Ludhiana

141004, Punjab, India

Full list of author information is available at the end of the article
}

\section{Springer Open}

(c) The Author(s). 2020 Open Access This article is licensed under a Creative Commons Attribution 4.0 International License, which permits use, sharing, adaptation, distribution and reproduction in any medium or format, as long as you give appropriate credit to the original author(s) and the source, provide a link to the Creative Commons licence, and indicate if changes were made. The images or other third party material in this article are included in the article's Creative Commons licence, unless indicated otherwise in a credit line to the material. If material is not included in the article's Creative Commons licence and your intended use is not permitted by statutory regulation or exceeds the permitted use, you will need to obtain permission directly from the copyright holder. To view a copy of this licence, visit http://creativecommons.org/licenses/by/4.0/. 
defoliation (Sayed et al. 2019). M. persicae can reduce photosynthetic capability of plant by producing sugary honeydew and affect the quality and quantity of crop (Frantz et al. 2004).

Management of the sucking insect pests is mainly by chemical insecticides but their excessive and indiscriminate use has led to insecticide resistance and residue problems apart from health hazards (Pilkington et al. 2010 and Pappas et al. 2013). Various parasitoids, predators, pathogens, and botanicals are being exploited for management of these pests (Ali et al. 2017 and Ullah et al. 2019). Management of insect pests using entomopathogenic fungi $(\mathrm{EPF})$ is regarded as an important alternative method for organic cultivation of vegetables under protected conditions(Manfrino et al. 2014 and Ali et al. 2018).

Entomopathogens are reported to control various crops insect pests. These EPF viz. Beauveria bassiana (Hypocreales: Cordycipitaceae), Metarhizium anisopliae (Hypocreales: Clavicipitaceae), Lecanicillium spp., (previously Verticillium lecanii) (Hypocreales: Cordycipitaceae), and Isaria fumosorosea (Hypocreales: Clavicipitaceae) (previously Paecilomyces fumosoroseus) are reported to kill insect by nutritional deficiency, tissue degradation, and release of toxins. The EPF contain cuticle degrading enzymes like protease; lipase and chitinase which degrade the insect cuticle, followed by penetration of fungal germ tube into insect body and thereby releases several mycotoxins such as Beauvericin, Beauverolides, and Bassianolide to kill the insect (Gabarty et al. 2014). The fungus initiates infection by adherence of conidia to the cuticle of a susceptible host by hydrophobic or enzymatic mechanisms and kills the host due to depletion of their hemolymph nutrients. In modern era, with the increasing awareness about the safety and quality of foods, long-term sustainability of the system and accumulating evidences of being equally productive, the organic farming is gaining importance.

So, present study aimed to evaluate various mycoformulations along with botanical formulation Azadirachtin to manage $M$. persicae and B. tabaci on capsicum in organic production under protected cultivation.

\section{Materials and methods}

\section{Fungal culture}

One Lecanicillium lecanii isolate (MTCC 956) procured form Institute of Microbial Technology, Chandigarh, India, and one native isolate Beauveria bassiana (Bb-B1) were used in the present study. These isolates were grown and maintained on potato dextrose agar (PDA) (dextrose 2\%, potatoes infusion form $20 \%$, agar 5\%, and chloramphenicol $0.5 \%)$ and stored at refrigeration temperature till further use.

\section{Production of mycoformulations}

These fungal isolates were inoculated and incubated on sterilized broken rice grains for 15 days at $25 \pm 2{ }^{\circ} \mathrm{C}$ and formulated to talc formulation according to methodology of Kaur and Joshi (2014).

\section{Test biopesticides}

Three entomopathogenic commercial mycoformulations viz. Bio-Catch ( $L$. lecanii $1.50 \%$ liquid formulation), Biomagic ( $M$. anisopliae $1.50 \%$ liquid formulation), Biopower (B. bassiana $1.50 \%$ liquid formulation) manufactured by T. Stanes \& Company Limited, Tamil Nadu, India), and one botanical formulation Azadirachtin (Econeem plus(1.0\%) from Margo Private Limited, Bengaluru and chemical malathion 50 EC (tusk) from Shivalik Company, Chandigarh, India, were used for the present study.

\section{Experiment method}

Capsicum crop was raised in polyhouse as per recommendation of Punjab Agricultural University Packages and Practices of Vegetable Crops. The capsicum hybrid variety "Indra" was transplanted on raised beds of $1.5 \mathrm{~m}$ width, with plant to plant and row to row spacing of 30 and $90 \mathrm{~cm}$, respectively. The experiment for the management of M. persicae and B. tabaci on capsicum was conducted for 2 years. The pre-treatment data of the aphid and whitefly population was recorded from 3 leaves (top, middle, and bottom) per plant and 3 foliar applications of all mycoformulations $(8,10$, and $12 \mathrm{~g} / \mathrm{l})$; Azadirachtin (@ 4 and $5 \mathrm{ml} / \mathrm{l}$ ) were given at 10 days interval in the evening hours. The experiment was conducted in randomized block design (RBD) with each concentration of biopesticide as one treatment with 3 replications and 5 plants per replica. Apart from these biorational treatments, one chemical malathion $50 \mathrm{EC}(4 \mathrm{ml} / \mathrm{l})$ and untreated check was also maintained to compare the efficacy of treatments.

\section{Aphid and whitefly counts}

The aphid and whitefly populations were recorded from the randomly selected 3 leaves from top, middle, and bottom canopy of randomly selected plant before treatment and after 3, 7, and 10 days of spray, respectively. To compare the efficacy of different EPF, percent reduction in the population of the aphid and whitefly over control was calculated, using (Henderson and Tilton's 1955) formula.

$$
\text { Corrected Mortality } \%=\left(1-\frac{\mathrm{n} \text { in Co before treatment } * \mathrm{n} \text { in T after treatment }}{\mathrm{n} \text { in Co after treatment } * \mathrm{n} \text { in T before treatment }}\right) * 100
$$

where $n=$ insect population, $T=$ treated, and Co $=$ control. 


\section{Fruit yield}

Capsicum fruits were harvested at regular interval and recorded for weight of fruits. Total yield was calculated in tons per hectare.

\section{Statistical analysis}

One-way ANOVA was conducted for all parameters, and means were compared by Duncan multiple range test (DMRT) at 5\% level of significance using (SPSS

Table 1 Population dynamics of aphid, Myzus persicae (Sulzer) on capsicum plant treated with indigenous and commercial bioformulations under protected cultivation $(2017,2018)$

\begin{tabular}{|c|c|c|c|c|c|c|c|c|c|c|c|c|}
\hline \multirow[t]{3}{*}{ Treatments } & \multirow{3}{*}{$\begin{array}{l}\text { Dose } \\
\text { (g or } \\
\mathrm{ml} / \mathrm{l})\end{array}$} & \multicolumn{10}{|c|}{ No. of aphid nymphs/plant*(mean \pm SE) } & \multirow{3}{*}{$\begin{array}{l}\text { Fruit } \\
\text { yield } \\
\text { (t/ha) }\end{array}$} \\
\hline & & \multirow{2}{*}{$\begin{array}{l}\text { Before } \\
\text { spray }\end{array}$} & \multicolumn{3}{|l|}{ I spray } & \multicolumn{3}{|l|}{ II spray } & \multicolumn{3}{|l|}{ III spray } & \\
\hline & & & 3DAS & 7DAS & 10DAS & 3DAS & 7DAS & 10DAS & 3DAS & 7DAS & 10DAS & \\
\hline \multirow{3}{*}{$\begin{array}{l}\text { Beauveria } \\
\text { bassiana } \\
\text { Bb-B1 }\end{array}$} & 8 & $\begin{array}{l}39.3 \pm \\
0.4^{a}\end{array}$ & $\begin{array}{l}36.0 \pm \\
0.6^{c}\end{array}$ & $\begin{array}{l}34.3^{ \pm \pm} \\
0.6^{\mathrm{e}}\end{array}$ & $\begin{array}{l}33.6 \pm \\
0.9^{d}\end{array}$ & $\begin{array}{l}31.2^{2 \pm} \\
1.2^{c}\end{array}$ & $\begin{array}{l}29.5 \pm \\
1.5^{c}\end{array}$ & $28.5 \pm 1.7^{\mathrm{e}}$ & $\begin{array}{l}26.0 \pm \\
2.5^{e}\end{array}$ & $\begin{array}{l}24.7 \pm \\
2.7^{\mathrm{e}}\end{array}$ & $\begin{array}{l}23.7 \pm \\
2.4^{e}\end{array}$ & $\begin{array}{l}42.1 \pm \\
1.1^{\mathrm{cd}}\end{array}$ \\
\hline & 10 & $\begin{array}{l}39.8 \pm \\
1.4^{\mathrm{a}}\end{array}$ & $\begin{array}{l}35.8 \pm \\
0.2^{\mathrm{bc}}\end{array}$ & $\begin{array}{l}34.3 \pm \\
0.2^{\mathrm{e}}\end{array}$ & $\begin{array}{l}33.6 \pm \\
0.4^{d}\end{array}$ & $\begin{array}{l}31.2 \pm \\
0.4^{c}\end{array}$ & $\begin{array}{l}29.3 \pm \\
0.4^{c}\end{array}$ & $\begin{array}{l}28.2 \pm \\
0.6^{\mathrm{de}}\end{array}$ & $\begin{array}{l}25.7 \pm \\
0.7^{\mathrm{e}}\end{array}$ & $\begin{array}{l}24.5 \pm \\
1.0^{\mathrm{e}}\end{array}$ & $\begin{array}{l}23.5 \pm \\
1.1^{e}\end{array}$ & $\begin{array}{l}42.4 \pm \\
0.9^{b c d}\end{array}$ \\
\hline & 12 & $\begin{array}{l}40.0 \pm \\
2.2^{\mathrm{a}}\end{array}$ & $\begin{array}{l}35.5 \pm \\
1.3^{\mathrm{bc}}\end{array}$ & $\begin{array}{l}34.0 \pm \\
1.1^{e}\end{array}$ & $\begin{array}{l}33.3 \pm \\
0.9^{d}\end{array}$ & $\begin{array}{l}30.7 \pm \\
0.8^{c}\end{array}$ & $\begin{array}{l}29.0 \pm \\
0.8^{c}\end{array}$ & $\begin{array}{l}28.0 \pm \\
1.0^{\mathrm{de}}\end{array}$ & $\begin{array}{l}25.5 \pm \\
1.7^{\mathrm{e}}\end{array}$ & $\begin{array}{l}24.2 \pm \\
1.9^{\mathrm{de}}\end{array}$ & $\begin{array}{l}23.0 \pm \\
1.7^{\mathrm{de}}\end{array}$ & $\begin{array}{l}42.8 \pm \\
0.9^{b c d}\end{array}$ \\
\hline \multirow{3}{*}{$\begin{array}{l}\text { Lecanicillium } \\
\text { lecanii } \\
\text { MTCC } 956\end{array}$} & 8 & $\begin{array}{l}38.9 \pm \\
0.6^{\mathrm{a}}\end{array}$ & $\begin{array}{l}33.7 \pm \\
2.1^{\mathrm{abc}}\end{array}$ & $\begin{array}{l}31.5 \pm \\
2.5^{b c d}\end{array}$ & $\begin{array}{l}30.2 \pm \\
2.4^{\mathrm{bcd}}\end{array}$ & $\begin{array}{l}26.7 \pm \\
3.9^{b c}\end{array}$ & $\begin{array}{l}24.3 \pm \\
4.5^{\mathrm{abc}}\end{array}$ & $\begin{array}{l}22.3 \pm \\
4.3^{\text {abcde }}\end{array}$ & $\begin{array}{l}18.2 \pm \\
3.5^{\mathrm{abcd}}\end{array}$ & $\begin{array}{l}16.0 \pm \\
4.0^{\mathrm{abcd}}\end{array}$ & $\begin{array}{l}15.2 \pm \\
3.6^{\text {bcde }}\end{array}$ & $\begin{array}{l}42.6 \pm \\
0.6^{b c d}\end{array}$ \\
\hline & 10 & $\begin{array}{l}38.4 \pm \\
1.5^{\mathrm{a}}\end{array}$ & $\begin{array}{l}32.7 \pm \\
2.2^{\mathrm{abc}}\end{array}$ & $\begin{array}{l}30.8 \pm \\
2.3^{\mathrm{abcd}}\end{array}$ & $\begin{array}{l}29.5 \pm \\
2.2^{\mathrm{bcd}}\end{array}$ & $\begin{array}{l}26.2 \pm \\
3.2^{\mathrm{abc}}\end{array}$ & $\begin{array}{l}23.7 \pm \\
3.7^{\mathrm{abc}}\end{array}$ & $\begin{array}{l}21.7 \pm \\
3.7^{\text {abcde }}\end{array}$ & $\begin{array}{l}17.7 \pm \\
3.2^{\mathrm{abcd}}\end{array}$ & $\begin{array}{l}15.5 \pm \\
3.8^{\mathrm{abc}}\end{array}$ & $\begin{array}{l}14.7 \pm \\
4.5^{\mathrm{abcd}}\end{array}$ & $\begin{array}{l}42.9 \pm \\
1.1^{b c d}\end{array}$ \\
\hline & 12 & $\begin{array}{l}38.7 \pm \\
0.9^{\mathrm{a}}\end{array}$ & $\begin{array}{l}32.5 \pm \\
2.5^{\mathrm{abc}}\end{array}$ & $\begin{array}{l}30.2 \pm \\
3.3^{\mathrm{abcd}}\end{array}$ & $\begin{array}{l}29.0 \pm \\
3.5^{\mathrm{abcd}}\end{array}$ & $\begin{array}{l}25.7 \pm \\
3.5^{\mathrm{abc}}\end{array}$ & $\begin{array}{l}23.2 \pm \\
3.7^{\mathrm{abc}}\end{array}$ & $\begin{array}{l}21.2 \pm \\
3.2^{\mathrm{abcd}}\end{array}$ & $\begin{array}{l}16.8 \pm \\
3.2^{\mathrm{abcd}}\end{array}$ & $\begin{array}{l}15.0 \pm \\
3.7^{\mathrm{abc}}\end{array}$ & $\begin{array}{l}14.3 \pm \\
4.9^{\mathrm{abc}}\end{array}$ & $\begin{array}{l}43.3 \pm \\
0.7^{b c d}\end{array}$ \\
\hline \multirow{3}{*}{$\begin{array}{l}\text { Beauveria } \\
\text { bassiana } \\
\text { Commercial } \\
\text { formulation }\end{array}$} & 8 & $\begin{array}{l}40.3 \pm \\
0.5^{a}\end{array}$ & $\begin{array}{l}35.5 \pm \\
0.9^{b c}\end{array}$ & $\begin{array}{l}33.5 \pm \\
0.9^{c d}\end{array}$ & $\begin{array}{l}32.3 \pm \\
0.9^{c d}\end{array}$ & $\begin{array}{l}28.8 \pm \\
1.3^{b c}\end{array}$ & $\begin{array}{l}27.0 \pm \\
1.7^{b c}\end{array}$ & $\begin{array}{l}25.7 \pm \\
1.4^{\text {cde }}\end{array}$ & $\begin{array}{l}21.7 \pm \\
1.2^{\mathrm{de}}\end{array}$ & $\begin{array}{l}19.5 \pm \\
1.3^{\text {bcde }}\end{array}$ & $\begin{array}{l}18.2 \pm \\
1.2^{\text {cde }}\end{array}$ & $\begin{array}{l}41.9 \pm \\
0.2^{\mathrm{cd}}\end{array}$ \\
\hline & 10 & $\begin{array}{l}39.5 \pm \\
0.9^{\mathrm{a}}\end{array}$ & $\begin{array}{l}34.3 \pm \\
0.4^{b c}\end{array}$ & $\begin{array}{l}32.3 \pm \\
0.7^{b c d}\end{array}$ & $\begin{array}{l}31.2 \pm \\
0.7^{b c d}\end{array}$ & $\begin{array}{l}27.7 \pm \\
1.8^{b c}\end{array}$ & $\begin{array}{l}25.8 \pm \\
1.9^{b c}\end{array}$ & $\begin{array}{l}24.5 \pm \\
1.75^{\mathrm{bcde}}\end{array}$ & $\begin{array}{l}20.7 \pm \\
1.4^{\text {bcde }}\end{array}$ & $\begin{array}{l}18.5 \pm \\
1.5^{\mathrm{bcde}}\end{array}$ & $\begin{array}{l}17.2 \pm \\
1.6^{\text {bcde }}\end{array}$ & $\begin{array}{l}42.5 \pm \\
2.8^{b c d}\end{array}$ \\
\hline & 12 & $\begin{array}{l}39.5 \pm \\
2.2^{\mathrm{a}}\end{array}$ & $\begin{array}{l}34.0 \pm \\
1.7^{\mathrm{abc}}\end{array}$ & $\begin{array}{l}31.8 \pm \\
1.6^{\mathrm{bcd}}\end{array}$ & $\begin{array}{l}30.7 \pm \\
1.2^{\text {bcd }}\end{array}$ & $\begin{array}{l}27.3 \pm \\
2.0^{b c}\end{array}$ & $\begin{array}{l}25.5 \pm \\
2.0^{\mathrm{bc}}\end{array}$ & $\begin{array}{l}24.2 \pm \\
2.04^{\mathrm{bcde}}\end{array}$ & $\begin{array}{l}20.3 \pm \\
1.9^{\text {bcde }}\end{array}$ & $\begin{array}{l}18.2 \pm \\
2.2^{\text {bcde }}\end{array}$ & $\begin{array}{l}16.8 \pm \\
2.0^{\text {bcde }}\end{array}$ & $\begin{array}{l}42.4 \pm \\
2.1^{b c d}\end{array}$ \\
\hline \multirow{3}{*}{$\begin{array}{l}\text { Metarhizium } \\
\text { anisopliae } \\
\text { Commercial } \\
\text { formulation }\end{array}$} & 8 & $\begin{array}{l}40.8 \pm \\
1.9^{\mathrm{a}}\end{array}$ & $\begin{array}{l}36.0 \pm \\
1.3^{c}\end{array}$ & $\begin{array}{l}33.8 \pm \\
0.9^{c d}\end{array}$ & $\begin{array}{l}32.5 \pm \\
0.8^{d}\end{array}$ & $\begin{array}{l}29.0 \pm \\
1.0^{b c}\end{array}$ & $\begin{array}{l}26.8 \pm \\
0.4^{b c}\end{array}$ & $\begin{array}{l}25.3 \pm \\
0.6^{\text {cde }}\end{array}$ & $\begin{array}{l}22.0 \pm \\
1.6^{\mathrm{de}}\end{array}$ & $\begin{array}{l}20.0 \pm \\
2.1^{\text {cde }}\end{array}$ & $\begin{array}{l}19.0 \pm \\
2.4^{\text {cde }}\end{array}$ & $\begin{array}{l}42.3 \pm \\
0.8^{b c d}\end{array}$ \\
\hline & 10 & $\begin{array}{l}40.2 \pm \\
2.1^{a}\end{array}$ & $\begin{array}{l}35.2 \pm \\
2.1^{\mathrm{bc}}\end{array}$ & $\begin{array}{l}32.8 \pm \\
2.2^{\text {bcd }}\end{array}$ & $\begin{array}{l}31.5 \pm \\
2.2^{\mathrm{bcd}}\end{array}$ & $\begin{array}{l}28.0 \pm \\
2.1^{b c}\end{array}$ & $\begin{array}{l}25.8 \pm \\
2.2^{\mathrm{bc}}\end{array}$ & $\begin{array}{l}24.3 \pm \\
2.4^{\text {bcde }}\end{array}$ & $\begin{array}{l}20.8 \pm \\
2.7^{\text {cde }}\end{array}$ & $\begin{array}{l}18.8 \pm \\
2.8^{\text {bcde }}\end{array}$ & $\begin{array}{l}17.8 \pm \\
2.8^{\text {bcde }}\end{array}$ & $\begin{array}{l}42.2 \pm \\
2.2^{\text {bcd }}\end{array}$ \\
\hline & 12 & $\begin{array}{l}39.8 \pm \\
0.7^{\mathrm{a}}\end{array}$ & $\begin{array}{l}34.2 \pm \\
1.6^{\mathrm{abc}}\end{array}$ & $\begin{array}{l}32.0 \pm \\
1.5^{\mathrm{bcd}}\end{array}$ & $\begin{array}{l}30.7 \pm \\
1.6^{\mathrm{bcd}}\end{array}$ & $\begin{array}{l}27.0 \pm \\
1.2^{\mathrm{bc}}\end{array}$ & $\begin{array}{l}24.8 \pm \\
1.4^{\mathrm{bc}}\end{array}$ & $\begin{array}{l}23.3 \pm \\
1.4^{\text {bcde }}\end{array}$ & $\begin{array}{l}19.8 \pm \\
0.7^{\text {bcde }}\end{array}$ & $\begin{array}{l}17.7 \pm \\
0.6^{\text {bcde }}\end{array}$ & $\begin{array}{l}17.0 \pm \\
0.9^{\text {bcde }}\end{array}$ & $\begin{array}{l}42.6 \pm \\
1.3^{b c d}\end{array}$ \\
\hline \multirow{3}{*}{$\begin{array}{l}\text { Lecanicillium } \\
\text { lecanii } \\
\text { Commercial } \\
\text { formulation }\end{array}$} & 8 & $\begin{array}{l}39.1 \pm \\
1.0^{\mathrm{a}}\end{array}$ & $\begin{array}{l}33.7 \pm \\
0.7^{\mathrm{abc}}\end{array}$ & $\begin{array}{l}31.8 \pm \\
0.3^{\text {bcd }}\end{array}$ & $\begin{array}{l}30.7 \pm \\
0.4^{\mathrm{bcd}}\end{array}$ & $\begin{array}{l}26.5 \pm \\
0.8^{\mathrm{abc}}\end{array}$ & $\begin{array}{l}24.2 \pm \\
0.7^{\mathrm{abc}}\end{array}$ & $\begin{array}{l}22.8 \pm \\
1.0^{\mathrm{abcde}}\end{array}$ & $\begin{array}{l}18.7 \pm \\
0.9^{\mathrm{bcd}}\end{array}$ & $\begin{array}{l}16.7 \pm \\
1.7^{\text {abcde }}\end{array}$ & $\begin{array}{l}16.0 \pm \\
1.5^{\text {bcde }}\end{array}$ & $\begin{array}{l}42.3 \pm \\
1.1^{b c d}\end{array}$ \\
\hline & 10 & $\begin{array}{l}39.2 \pm \\
2.6^{\mathrm{a}}\end{array}$ & $\begin{array}{l}33.3 \pm \\
1.6^{\mathrm{abc}}\end{array}$ & $\begin{array}{l}31.5 \pm \\
1.7^{\text {bcd }}\end{array}$ & $\begin{array}{l}30.2 \pm \\
1.9^{\mathrm{bcd}}\end{array}$ & $\begin{array}{l}26.0 \pm \\
3.0^{\mathrm{abc}}\end{array}$ & $\begin{array}{l}23.5 \pm \\
3.0^{\mathrm{abc}}\end{array}$ & $\begin{array}{l}22.3 \pm \\
3.1^{\mathrm{abcde}}\end{array}$ & $\begin{array}{l}18.0 \pm \\
3.0^{\mathrm{abcd}}\end{array}$ & $\begin{array}{l}16.0 \pm \\
4.1^{\mathrm{abcd}}\end{array}$ & $\begin{array}{l}15.5 \pm \\
3.3^{\text {bcde }}\end{array}$ & $\begin{array}{l}43.1 \pm \\
1.0^{\text {bcd }}\end{array}$ \\
\hline & 12 & $\begin{array}{l}39.0 \pm \\
1.0^{\mathrm{a}}\end{array}$ & $\begin{array}{l}33.0 \pm \\
1.2^{\mathrm{abc}}\end{array}$ & $\begin{array}{l}31.0 \pm \\
1.5^{\text {bcd }}\end{array}$ & $\begin{array}{l}29.8 \pm \\
1.6^{\mathrm{bcd}}\end{array}$ & $\begin{array}{l}25.5 \pm \\
1.3^{\mathrm{abc}}\end{array}$ & $\begin{array}{l}23.0 \pm \\
2.0^{\mathrm{abc}}\end{array}$ & $\begin{array}{l}21.8 \pm \\
1.8^{\text {abcde }}\end{array}$ & $\begin{array}{l}17.7 \pm \\
2.2^{\mathrm{abcd}}\end{array}$ & $\begin{array}{l}15.8 \pm \\
3.1^{\mathrm{abcd}}\end{array}$ & $\begin{array}{l}15.2 \pm \\
2.7^{\text {bcde }}\end{array}$ & $\begin{array}{l}42.9 \pm \\
1.0^{b c d}\end{array}$ \\
\hline \multirow[t]{2}{*}{$\begin{array}{l}\text { Azadirachtin } 1 \% \\
(10,000 \text { ppm) }\end{array}$} & 4 & $\begin{array}{l}38.3 \pm \\
1.5^{a}\end{array}$ & $\begin{array}{l}30.8 \pm \\
1.3^{\mathrm{abc}}\end{array}$ & $\begin{array}{l}28.5 \pm \\
1.0^{\mathrm{abc}}\end{array}$ & $\begin{array}{l}27.0 \pm \\
1.2^{\mathrm{abc}}\end{array}$ & $\begin{array}{l}23.2 \pm \\
1.0^{\mathrm{ab}}\end{array}$ & $\begin{array}{l}20.3 \pm \\
0.7^{\mathrm{ab}}\end{array}$ & $\begin{array}{l}18.7 \pm \\
0.7^{\mathrm{abc}}\end{array}$ & $\begin{array}{l}14.3 \pm \\
0.7^{\mathrm{abc}}\end{array}$ & $\begin{array}{l}12.2 \pm \\
0.3^{\mathrm{abc}}\end{array}$ & $\begin{array}{l}10.7 \pm \\
0.4^{\mathrm{abc}}\end{array}$ & $\begin{array}{l}46.2 \pm \\
2.0^{\mathrm{abc}}\end{array}$ \\
\hline & 5 & $\begin{array}{l}38.8 \pm \\
1.4^{\mathrm{a}}\end{array}$ & $\begin{array}{l}30.7 \pm \\
1.1^{\mathrm{ab}}\end{array}$ & $\begin{array}{l}27.8 \pm \\
0.9^{\mathrm{ab}}\end{array}$ & $\begin{array}{l}26.5 \pm \\
1.1^{a b}\end{array}$ & $\begin{array}{l}22.7 \pm \\
1.6^{\mathrm{ab}}\end{array}$ & $\begin{array}{l}19.8 \pm \\
1.2^{\mathrm{ab}}\end{array}$ & $\begin{array}{l}18.2 \pm \\
1.1^{\mathrm{ab}}\end{array}$ & $\begin{array}{l}13.8 \pm \\
1.2^{\mathrm{ab}}\end{array}$ & $\begin{array}{l}11.5 \pm \\
1.0^{\mathrm{ab}}\end{array}$ & $\begin{array}{l}9.5 \pm \\
0.8^{\mathrm{ab}}\end{array}$ & $\begin{array}{l}47.1 \pm \\
1.6^{\mathrm{ab}}\end{array}$ \\
\hline $\begin{array}{l}\text { Chemical } \\
\text { check } \\
\text { (malathion } \\
50 \text { EC) }\end{array}$ & 4 & $\begin{array}{l}38.3 \pm \\
1.2^{\mathrm{a}}\end{array}$ & $\begin{array}{l}29.2 \pm \\
0.7^{a}\end{array}$ & $\begin{array}{l}25.8 \pm \\
1.0^{a}\end{array}$ & $\begin{array}{l}24.2 \pm \\
0.9^{a}\end{array}$ & $\begin{array}{l}19.8 \pm \\
1.0^{\mathrm{a}}\end{array}$ & $\begin{array}{l}17.2 \pm \\
0.7^{a}\end{array}$ & $16.0 \pm 0.3^{\mathrm{a}}$ & $\begin{array}{l}11.7 \pm \\
0.4^{\mathrm{a}}\end{array}$ & $9.3 \pm 0.4^{\mathrm{a}}$ & $6.7 \pm 0.8^{\mathrm{a}}$ & $\begin{array}{l}49.4 \pm \\
0.8^{\mathrm{a}}\end{array}$ \\
\hline Control & & $\begin{array}{l}37.6 \pm \\
3.6^{a}\end{array}$ & $\begin{array}{l}41.1 \pm \\
2.2^{d}\end{array}$ & $\begin{array}{l}39.3 \pm \\
2.0^{f}\end{array}$ & $\begin{array}{l}39.3 \pm \\
1.6^{\mathrm{e}}\end{array}$ & $\begin{array}{l}39.0 \pm \\
2.6^{d}\end{array}$ & $\begin{array}{l}37.0 \pm \\
2.2^{d}\end{array}$ & $37.5 \pm 1.0^{f}$ & $\begin{array}{l}37.2 \pm \\
1.9^{f}\end{array}$ & $37.2 \pm 3.0^{f}$ & $\begin{array}{l}36.3 \pm \\
3.2^{f}\end{array}$ & $\begin{array}{l}39.8 \pm \\
2.0^{d}\end{array}$ \\
\hline$F_{(18,38) \text { values }}$ & & 0.23 & 2.9 & 3.3 & 4.0 & 3.8 & 3.8 & 5.0 & 7.5 & 6.2 & 6.3 & 2.2 \\
\hline$P$ & & 1.00 & 0.03 & $<0.01$ & $<0.001$ & $<0.001$ & $<0.001$ & $<0.001$ & $<0.001$ & $<0.001$ & $<0.001$ & 0.022 \\
\hline
\end{tabular}

*Values represent means of 3 replicates

DAS days after spray

Means within each column bearing different letters are significantly different according to the Duncan test $(P=0.05)$ 
Table 2 Percentage reduction of aphid, Myzus persicae, on capsicum plant treated with indigenous and commercial bioformulations under protected cultivation $(2017,2018)$

\begin{tabular}{|c|c|c|c|c|c|c|c|c|c|c|}
\hline \multirow[t]{3}{*}{ Treatments } & \multirow{3}{*}{$\begin{array}{l}\text { Dose } \\
\text { (g or } \\
\mathrm{ml} / \mathrm{l} \text { ) }\end{array}$} & \multicolumn{9}{|c|}{ Percent reduction over control *(mean \pm SE) } \\
\hline & & \multicolumn{3}{|l|}{ spray } & \multicolumn{3}{|l|}{ II spray } & \multicolumn{3}{|l|}{ III spray } \\
\hline & & 3DAS & 7DAS & 10DAS & 3DAS & 7DAS & 10DAS & 3DAS & 7DAS & 10DAS \\
\hline \multirow[t]{3}{*}{$\begin{array}{l}\text { Beauveria bassiana } \\
\mathrm{Bb}-\mathrm{B} 1\end{array}$} & 8 & $\begin{array}{l}16.3 \pm \\
3.9^{\mathrm{a}}\end{array}$ & $\begin{array}{l}16.6 \pm \\
4.3^{b}\end{array}$ & $\begin{array}{l}18.4 \pm \\
5.0^{\mathrm{b}}\end{array}$ & $23.7 \pm 6.2^{c}$ & $23.9 \pm 4.9^{c}$ & $27.4 \pm 2.5^{\mathrm{e}}$ & $33.2 \pm 1.4^{e}$ & $36.6 \pm 3.9^{e}$ & $37.8 \pm 4.7^{e}$ \\
\hline & 10 & $\begin{array}{l}17.7 \pm \\
7.6^{\mathrm{a}}\end{array}$ & $\begin{array}{l}17.7 \pm \\
7.9^{b}\end{array}$ & $\begin{array}{l}19.4 \pm \\
8.5^{b}\end{array}$ & $24.6 \pm 9.4^{c}$ & $25.2 \pm 8.8^{c}$ & $29.2 \pm 8.0^{\mathrm{e}}$ & $34.9 \pm 5.8^{e}$ & $37.8 \pm 8.0^{e}$ & $39.0 \pm 7.1^{\mathrm{e}}$ \\
\hline & 12 & $\begin{array}{l}18.8 \pm \\
6.2^{\mathrm{a}}\end{array}$ & $\begin{array}{l}18.8 \pm \\
6.3^{b}\end{array}$ & $\begin{array}{l}20.5 \pm \\
7.4^{b}\end{array}$ & $26.2 \pm 8.4^{c}$ & $26.4 \pm 7.5^{c}$ & $\begin{array}{l}30.0 \pm \\
5.2^{\mathrm{de}}\end{array}$ & $35.6 \pm 3.1^{e}$ & $\begin{array}{l}38.9 \pm \\
5.9^{\mathrm{de}}\end{array}$ & $40.5 \pm 5.8^{\text {de }}$ \\
\hline \multirow[t]{3}{*}{$\begin{array}{l}\text { Lecanicillium lecanii } \\
\text { MTCC } 956\end{array}$} & 8 & $\begin{array}{l}21.0 \pm \\
0.5^{\mathrm{a}}\end{array}$ & $\begin{array}{l}22.6 \pm \\
2.1^{\mathrm{ab}}\end{array}$ & $\begin{array}{l}25.9 \pm \\
1.2^{\mathrm{ab}}\end{array}$ & $\begin{array}{l}34.0 \pm \\
5.11^{\mathrm{abc}}\end{array}$ & $\begin{array}{l}36.5 \pm \\
7.6^{b c}\end{array}$ & $\begin{array}{l}42.5 \pm \\
6.3^{\mathrm{abcd}}\end{array}$ & $\begin{array}{l}52.8 \pm \\
5.8^{\text {abcd }}\end{array}$ & $\begin{array}{l}58.4 \pm \\
5.4^{\mathrm{ab} c d}\end{array}$ & $\begin{array}{l}59.7 \pm \\
5.4^{\mathrm{abcd}}\end{array}$ \\
\hline & 10 & $\begin{array}{l}22.3 \pm \\
2.7^{\mathrm{a}}\end{array}$ & $\begin{array}{l}23.4 \pm \\
4.4^{\mathrm{ab}}\end{array}$ & $\begin{array}{l}26.7 \pm \\
5.1^{\mathrm{ab}}\end{array}$ & $\begin{array}{l}34.4 \pm \\
6.4^{\mathrm{abc}}\end{array}$ & $\begin{array}{l}37.5 \pm \\
6.2^{\mathrm{abc}}\end{array}$ & $\begin{array}{l}43.5 \pm \\
3.1^{\mathrm{abc}}\end{array}$ & $\begin{array}{l}53.6 \pm \\
3.0^{\mathrm{abcd}}\end{array}$ & $\begin{array}{l}59.3 \pm \\
2.5^{\mathrm{abc}}\end{array}$ & $\begin{array}{l}60.6 \pm \\
5.4^{\mathrm{abc}}\end{array}$ \\
\hline & 12 & $\begin{array}{l}23.1 \pm \\
2.0^{\mathrm{a}}\end{array}$ & $\begin{array}{l}25.5 \pm \\
3.1^{\mathrm{ab}}\end{array}$ & $\begin{array}{l}28.4 \pm \\
2.5^{\mathrm{ab}}\end{array}$ & $\begin{array}{l}36.1 \pm \\
3.0^{\mathrm{abc}}\end{array}$ & $\begin{array}{l}39.2 \pm \\
5.0^{\mathrm{abc}}\end{array}$ & $\begin{array}{l}45.2 \pm \\
4.3^{\mathrm{abc}}\end{array}$ & $\begin{array}{l}56.0 \pm \\
5.0^{\mathrm{abcd}}\end{array}$ & $\begin{array}{l}60.8 \pm \\
4.5^{\mathrm{abc}}\end{array}$ & $\begin{array}{l}61.7 \pm \\
6.6^{\mathrm{abc}}\end{array}$ \\
\hline \multirow[t]{3}{*}{$\begin{array}{l}\text { Beauveria bassiana } \\
\text { Commercial formulation }\end{array}$} & 8 & $\begin{array}{l}19.4 \pm \\
3.2^{\mathrm{a}}\end{array}$ & $\begin{array}{l}20.6 \pm \\
3.5^{\mathrm{ab}}\end{array}$ & $\begin{array}{l}23.3 \pm \\
4.1^{\mathrm{ab}} \pm\end{array}$ & $\begin{array}{l}31.1 \pm \\
3.9^{b c}\end{array}$ & $\begin{array}{l}32.0 \pm \\
2.4^{b c}\end{array}$ & $\begin{array}{l}36.2 \pm \\
3.1^{1 \mathrm{de}}\end{array}$ & $\begin{array}{l}45.6 \pm \\
3.5^{\mathrm{cde}^{ \pm}}\end{array}$ & $\begin{array}{l}51.1 \pm \\
6.1^{1 \mathrm{de}}\end{array}$ & $\begin{array}{l}53.4 \pm \\
6.0^{\text {cde }}\end{array}$ \\
\hline & 10 & $\begin{array}{l}20.5 \pm \\
5.0^{\mathrm{a}} \pm\end{array}$ & $\begin{array}{l}21.8 \pm \\
4.4^{\mathrm{ab}}\end{array}$ & $\begin{array}{l}24.6 \pm \\
5.4^{\mathrm{ab}}\end{array}$ & $\begin{array}{l}32.5 \pm \\
3.2^{2 \mathrm{abc}}\end{array}$ & $\begin{array}{l}33.6 \pm \\
2.3^{\mathrm{bc}} \pm\end{array}$ & $\begin{array}{l}38.0 \pm \\
6.4^{4 d e}\end{array}$ & $\begin{array}{l}47.1 \pm \\
7.0^{\text {cde }}\end{array}$ & $\begin{array}{l}52.7 \pm \pm \\
8.2^{\text {bcde }}\end{array}$ & $\begin{array}{l}55.1 \pm \\
8.9^{\text {bcde }}\end{array}$ \\
\hline & 12 & $\begin{array}{l}21.3 \pm \\
3.3^{\mathrm{a}}\end{array}$ & $\begin{array}{l}23.0 \pm \\
3.7^{\mathrm{ab}}\end{array}$ & $\begin{array}{l}26.0 \pm \\
5.3^{\mathrm{ab}}\end{array}$ & $\begin{array}{l}33.4 \pm \\
2.5^{\mathrm{abc}}\end{array}$ & $\begin{array}{l}34.5 \pm \\
2.3^{\mathrm{bc}}\end{array}$ & $\begin{array}{l}39.0 \pm \\
5.6^{b c d e}\end{array}$ & $\begin{array}{l}48.0 \pm \\
7.1^{\text {bcde }}\end{array}$ & $\begin{array}{l}53.5 \pm \\
9.5^{\text {bcde }}\end{array}$ & $\begin{array}{l}56.0 \pm \\
9.5^{\text {bcde }}\end{array}$ \\
\hline \multirow[t]{3}{*}{$\begin{array}{l}\text { Metarhizium anisopliae } \\
\text { Commercial formulation }\end{array}$} & 8 & $\begin{array}{l}19.2 \pm \\
3.7^{\mathrm{a}}\end{array}$ & $\begin{array}{l}20.8 \pm \\
3.1^{\mathrm{ab}}\end{array}$ & $\begin{array}{l}24.0 \pm \\
4.0^{\mathrm{ab}}\end{array}$ & $\begin{array}{l}31.5 \pm \\
3.2^{b c}\end{array}$ & $\begin{array}{l}33.2 \pm \\
3.0^{b c}\end{array}$ & $\begin{array}{l}37.8 \pm \\
4.3^{\mathrm{cde}}\end{array}$ & $\begin{array}{l}45.5 \pm \\
6.8^{\mathrm{de}}\end{array}$ & $\begin{array}{l}50.4 \pm \\
9.2^{2 \mathrm{de}}\end{array}$ & $\begin{array}{l}51.8 \pm \\
10.8^{\text {cde }}\end{array}$ \\
\hline & 10 & $\begin{array}{l}20.0 \pm \\
3.5^{\mathrm{a}}\end{array}$ & $\begin{array}{l}22.0 \pm \\
3.6^{\mathrm{ab}}\end{array}$ & $\begin{array}{l}25.2 \pm \\
4.1^{\mathrm{ab}}\end{array}$ & $\begin{array}{l}33.0 \pm \\
4.4^{\mathrm{abc}}\end{array}$ & $\begin{array}{l}34.8 \pm \\
2.6^{b c}\end{array}$ & $\begin{array}{l}39.4 \pm \\
4.4^{\text {bcde }}\end{array}$ & $\begin{array}{l}47.7 \pm \\
5.4^{\text {bcde }}\end{array}$ & $\begin{array}{l}52.7 \pm \\
6.5^{\text {bcde }}\end{array}$ & $\begin{array}{l}54.2 \pm \\
7.1^{\text {bcde }}\end{array}$ \\
\hline & 12 & $\begin{array}{l}21.5 \pm \\
5.3^{\mathrm{a}}\end{array}$ & $\begin{array}{l}23.3 \pm \\
5.1^{\mathrm{ab}}\end{array}$ & $\begin{array}{l}26.5 \pm \\
6.4^{\mathrm{ab}}\end{array}$ & $\begin{array}{l}34.7 \pm \\
7.0^{\mathrm{abc}}\end{array}$ & $\begin{array}{l}36.7 \pm \\
7.0^{b c}\end{array}$ & $\begin{array}{l}41.3 \pm \\
5.2^{\text {bcde }}\end{array}$ & $\begin{array}{l}49.7 \pm \\
4.1^{b c d e}\end{array}$ & $\begin{array}{l}55.2 \pm \\
6.3^{\text {bcde }}\end{array}$ & $\begin{array}{l}55.9 \pm \\
5.3^{\text {bcde }}\end{array}$ \\
\hline \multirow[t]{3}{*}{$\begin{array}{l}\text { Lecanicillium lecanii } \\
\text { Commercial formulation }\end{array}$} & 8 & $\begin{array}{l}21.1 \pm \\
5.0^{\mathrm{a}}\end{array}$ & $\begin{array}{l}22.2 \pm \\
4.8^{\mathrm{ab}}\end{array}$ & $\begin{array}{l}25.0 \pm \\
5.3^{\mathrm{ab}}\end{array}$ & $\begin{array}{l}34.6 \pm \\
5.6^{\mathrm{abc}}\end{array}$ & $\begin{array}{l}37.2 \pm \\
4.1^{b c}\end{array}$ & $\begin{array}{l}41.4 \pm \\
2.5^{\text {bcde }}\end{array}$ & $\begin{array}{l}51.7 \pm \\
1.7^{b c d}\end{array}$ & $\begin{array}{l}56.9 \pm \\
2.0^{\text {bcde }}\end{array}$ & $\begin{array}{l}57.7 \pm \\
2.3^{\text {bcde }}\end{array}$ \\
\hline & 10 & $\begin{array}{l}22.1 \pm \\
4.4^{\mathrm{a}}\end{array}$ & $\begin{array}{l}23.2 \pm \\
4.2^{2 \mathrm{~b}}\end{array}$ & $\begin{array}{l}26.4 \pm \\
4.3^{\mathrm{ab}}\end{array}$ & $\begin{array}{l}36.1 \pm \\
3.2^{2 \mathrm{abc}}\end{array}$ & $\begin{array}{l}39.1 \pm \\
3.2^{\mathrm{abc}}\end{array}$ & $\begin{array}{l}42.9 \pm \\
2.0^{\mathrm{abcd}}\end{array}$ & $\begin{array}{l}53.6 \pm \\
0.9^{\text {abcd }}\end{array}$ & $\begin{array}{l}58.7 \pm \\
0.7^{\text {abcd }}\end{array}$ & $\begin{array}{l}59.1 \pm \\
1.1^{\mathrm{abcd}}\end{array}$ \\
\hline & 12 & $\begin{array}{l}22.6 \pm \\
3.2^{\mathrm{a}}\end{array}$ & $\begin{array}{l}24.1 \pm \\
2.6^{\mathrm{ab}}\end{array}$ & $\begin{array}{l}27.0 \pm \\
3.1^{\mathrm{ab}} \pm\end{array}$ & $\begin{array}{l}37.0 \pm \\
3.7^{\mathrm{abc}}\end{array}$ & $\begin{array}{l}40.1 \pm \pm \\
3.1^{\mathrm{abc}}\end{array}$ & $\begin{array}{l}43.9 \pm \\
2.0^{\mathrm{abc}}\end{array}$ & $\begin{array}{l}54.2 \pm \\
1.4^{\text {abcd }}\end{array}$ & $\begin{array}{l}59.0 \pm \\
1.0^{\mathrm{abcd}}\end{array}$ & $\begin{array}{l}59.8 \pm \\
1.4^{\mathrm{abcd}}\end{array}$ \\
\hline \multirow[t]{2}{*}{$\begin{array}{l}\text { Azadirachtin } 1 \%(10,000 \\
\text { ppm) }\end{array}$} & 4 & $\begin{array}{l}26.4 \pm \\
4.1^{\mathrm{a}}\end{array}$ & $\begin{array}{l}29.0 \pm \\
4.0^{\mathrm{ab}}\end{array}$ & $\begin{array}{l}32.7 \pm \\
4.1^{a b}\end{array}$ & $\begin{array}{l}41.8 \pm \\
5.3^{\mathrm{abc}}\end{array}$ & $\begin{array}{l}46.2 \pm \\
4.2^{\mathrm{ab}}\end{array}$ & $\begin{array}{l}51.2 \pm \\
3.2^{\mathrm{abc}}\end{array}$ & $\begin{array}{l}62.2 \pm \\
5.4^{\mathrm{abc}}\end{array}$ & $\begin{array}{l}67.9 \pm \\
5.7^{\mathrm{abc}}\end{array}$ & $\begin{array}{l}71.2 \pm \\
4.8^{\mathrm{abc}}\end{array}$ \\
\hline & 5 & $\begin{array}{l}28.0 \pm \\
4.0^{\mathrm{a}}\end{array}$ & $\begin{array}{l}31.5 \pm \\
4.5^{5 \mathrm{~b}}\end{array}$ & $\begin{array}{l}34.8 \pm \\
4.2^{\mathrm{ab}}\end{array}$ & $\begin{array}{l}43.8 \pm \\
2.9^{\mathrm{ab}}\end{array}$ & $\begin{array}{l}48.2 \pm \\
2.9^{\mathrm{ab}}\end{array}$ & $\begin{array}{l}53.1 \pm \\
3.4^{\mathrm{ab}}\end{array}$ & $\begin{array}{l}64.0 \pm \\
5.3^{\mathrm{ab}}\end{array}$ & $\begin{array}{l}70.1 \pm \\
4.6^{\mathrm{ab}}\end{array}$ & $74.7 \pm 3.7^{\mathrm{ab}}$ \\
\hline $\begin{array}{l}\text { Chemical check (malathion } \\
50 \mathrm{EC} \text { ) }\end{array}$ & 4 & $\begin{array}{l}30.4 \pm \\
3.8^{\mathrm{a}}\end{array}$ & $\begin{array}{l}35.6 \pm \\
5.1^{\mathrm{a}}\end{array}$ & $\begin{array}{l}39.8 \pm \\
5.5^{\mathrm{a}}\end{array}$ & $50.2 \pm 5.1^{\mathrm{a}}$ & $54.5 \pm 1.4^{\mathrm{a}}$ & $58.2 \pm 3.6^{a}$ & $69.2 \pm 4.7^{\mathrm{a}}$ & $75.4 \pm 3.3^{\mathrm{a}}$ & $82.0 \pm 4.9^{a}$ \\
\hline$F$ values & & 0.7 & 1.1 & 1.1 & 1.6 & 2.5 & 3.2 & 4.1 & 3.3 & 3.5 \\
\hline P & & 0.8 & 0.4 & 0.4 & 0.1 & 0.01 & 0.01 & 0.01 & 0.01 & 0.01 \\
\hline
\end{tabular}

Mean mortality (\%) \pm standard error of $M$. persicae recorded at different time intervals (DAS, days after spray) for bioassays performed with different commercial and indigenous strains

Treatment columns bearing different letters are significantly different from other treatments according to Duncan test $(P=0.05)$

${ }^{*}$ Calculated as per Henderson and Tilton (1955) formula

2015). In order to correct mortality data in the treatment with that in the control, Henderson and Tilton's (1955) formula was used.

\section{Results and discussion}

Biopesticides against $M$. persicae

Under protected cultivation, $M$. persicae was recorded on capsicum plant for 2 consecutive years, 2017 and
2018. The pooled data presented in Table 1 depicted that the aphid individuals in the treatment control increased and decreased slightly, with maximum population density (41.1 aphids per 3 leaves). After 10 days of 1 st spray, aphid individuals declined in all the mycoformulation treatments, 29.0-33.6 aphids per 3 leaves at the concentration of $1 \times 10^{8} \mathrm{cfu} / \mathrm{ml}$, and in Azadirachtin, 26.5-27.0 aphids per 3 leaves at 
concentration of $1 \%$. The aphid infestation decreased gradually throughout the experiment. Both commercial and indigenous bioformulation decreased the aphid individuals after 3rd spray. The aphid population in L. lecanii MTCC 956 at $12 \mathrm{~g} / \mathrm{l}$ was 16.8, 15.0, and 14.3 aphids per 3 leaves with 56.0, 60.8, and $61.7 \%$ population reduction after 3,7 , and 10 days after spray (DAS), respectively and was nonsignificant with MTCC 956 at $10 \mathrm{~g} / \mathrm{l}$ recorded 17.7, 15.5, and 14.7 aphids per 3 leaves with 53.6, 59.3, and $60.6 \%$ population reduction after 3,7 , and 10 DAS, respectively Table 2. Azadirachtin at 4 and $5 \mathrm{ml} / 1$ were non-significant with each other and with $L$. lecanii MTCC 956 formulation. Azadirachtin at $5 \mathrm{ml} / \mathrm{l}$ recorded aphid population 13.8, 11.5, and 9.5 with 64.0, 70.1, and $74.7 \%$ aphid population reduction after 3,7 , and 10 days after 3 sprays (Fig. 1). In the present studies, it was observed that Azadirachtin $1 \%$ at 4 and $5 \mathrm{ml} / \mathrm{l}$ and L. lecani bioformulation at 10 and 12 $\mathrm{g} / \mathrm{l}$ were significantly better than all other fungal formulations in management of the aphid. Highest fruit yield $(49.4 \mathrm{t} / \mathrm{ha})$ was in chemical check malathion 50 $\mathrm{EC}$ at $4 \mathrm{ml} / \mathrm{l}$ and was non-significant with yield recorded in Azadirachtin at $5 \mathrm{ml} / \mathrm{l}$ (47.1 t/ha) and $4 \mathrm{ml} / \mathrm{l}$ (46.2 $\mathrm{t} / \mathrm{ha}$ ). So, bioformulations of $L$. lecanii and azadirachtin $1 \%$ against $M$. persicae resulted in a significant increase in the mortality of $M$. persicae under protected cultivation and also no plant damage was recorded during the experiment. The high mortality recorded in L. lecanii may be due to fact that it germinated well under a wide range of temperatures and humidity and thus made this fungus more virulent, whereas, the Azadirachtin affected the reproductive rate of sucking insect pests causing more nymphal mortality and thus reducing their survival period and fecundity. Obtained results are in corroboration with $\mathrm{Vu}$ et al. (2007) who evaluated 12 strains of EPF viz., L. lecanii, P. farinosus, B. bassiana, M. anisopliae, Cordyceps scarabaeicola, and Nomuraea rileyi (Hypocreales: Clavicipitaceae) against aphids on cabbage and cucumber under greenhouse conditions and recorded that $L$. lecanii 41185 strain was highly virulent than all other strains against $M$. persicae and A. gossypii. They also reported that $L$. lecanii 41185 strain germinated and grew well under wide range of temperature and humidity. This finding coincides with the results of Mohammed et al. (2018) who recorded $L$. lecanii better than $M$. anisopliae and B. bassiana against $M$. persicae and $A$. gossypii under laboratory and greenhouse cultivations and in which they evaluated 4 procured native isolates of $B$. bassiana, $M$. anisopliae, L. lecanii, and Chaetomium globosum and recorded their efficacy against the aphids $M$. persicae and $A$. gossypii and showed that $L$. lecanii showed the highest mortality than all 3 isolates against $M$. persicae and $A$. gossypii under laboratory as well as under greenhouse cultivation when applied at the concentration of $1 \times 10^{8}$ conidia $/ \mathrm{ml}$. Santos et al.

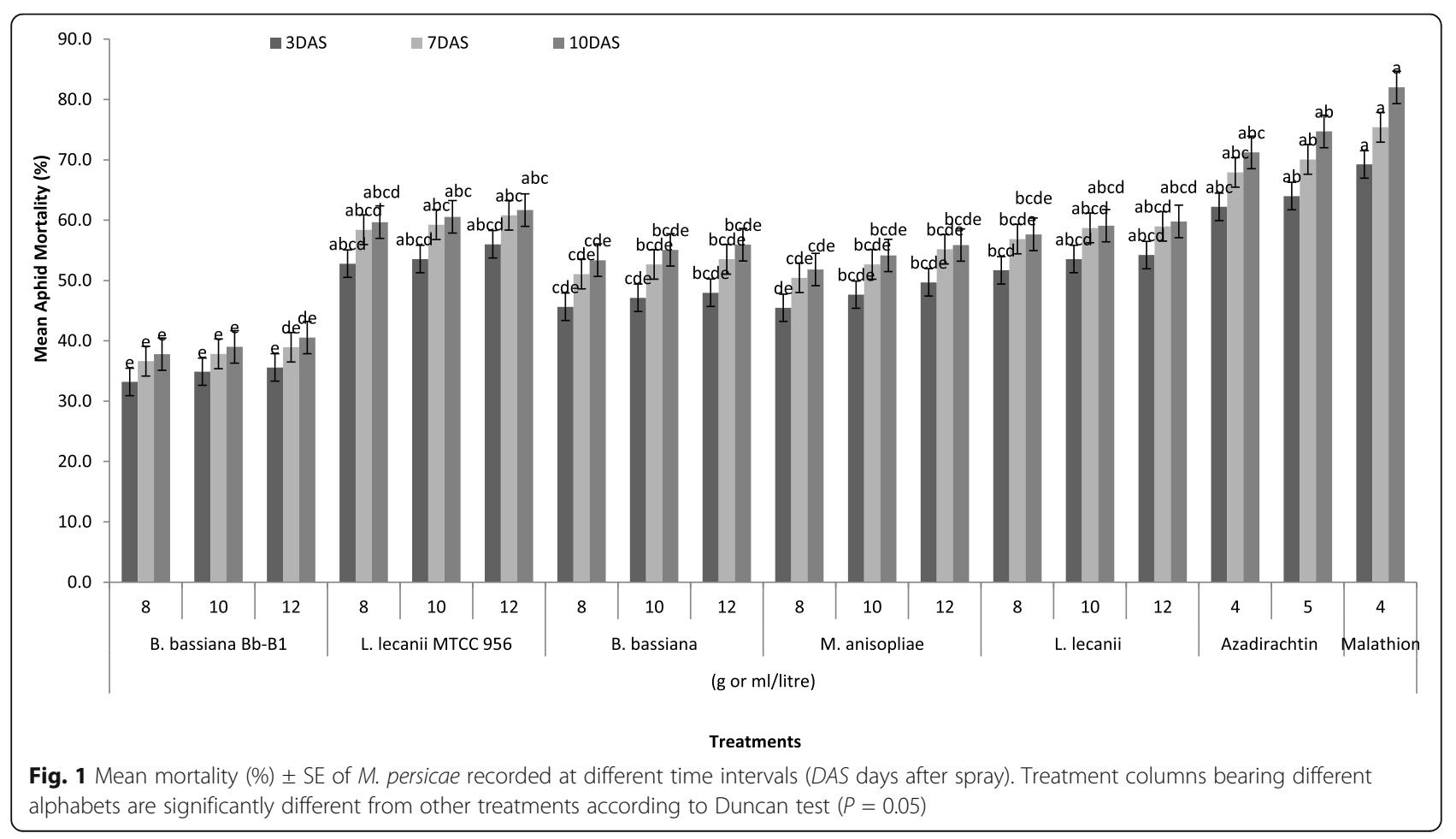


Table 3 Population dynamics of whitefly, Bemisia tabaci (Gennadius) on capsicum plant treated with indigenous and commercial bioformulations under protected cultivation $(2017,2018)$

\begin{tabular}{|c|c|c|c|c|c|c|c|c|c|c|c|c|}
\hline \multirow[t]{3}{*}{ Treatments } & \multirow{3}{*}{$\begin{array}{l}\text { Dose } \\
\text { (g or } \\
\mathrm{ml} / \mathrm{l})\end{array}$} & \multicolumn{10}{|c|}{ No. of whitefly adults/plant*(mean \pm SE) } & \multirow{3}{*}{$\begin{array}{l}\text { Fruit } \\
\text { yield (t/ } \\
\text { ha) }\end{array}$} \\
\hline & & \multirow{2}{*}{$\begin{array}{l}\text { Before } \\
\text { spray }\end{array}$} & \multicolumn{3}{|l|}{ I spray } & \multicolumn{3}{|l|}{ II spray } & \multicolumn{3}{|l|}{ III spray } & \\
\hline & & & 3DAS & 7DAS & 10DAS & 3DAS & 7DAS & 10DAS & 3DAS & 7DAS & 10DAS & \\
\hline \multirow[t]{3}{*}{$\begin{array}{l}\text { Beauveria bassiana } \\
\text { Bb-B1 }\end{array}$} & 8 & $\begin{array}{l}22.8 \pm \\
2.9^{\mathrm{a}}\end{array}$ & $\begin{array}{l}20.5 \pm \\
1.4^{\mathrm{abc}}\end{array}$ & $\begin{array}{l}19.3 \pm \\
1.8^{\mathrm{ab}}\end{array}$ & $\begin{array}{l}18.7 \pm \\
2.0^{\mathrm{ab}}\end{array}$ & $\begin{array}{l}17.0 \pm \\
1.7^{\mathrm{bc}}\end{array}$ & $\begin{array}{l}16.0 \pm \\
1.7^{c}\end{array}$ & $\begin{array}{l}15.7 \pm \\
2.0^{d}\end{array}$ & $\begin{array}{l}14.0 \pm \\
2.3^{d}\end{array}$ & $\begin{array}{l}13.0 \pm \\
2.1^{d}\end{array}$ & $\begin{array}{l}12.3 \pm \\
2.0^{d}\end{array}$ & $\begin{array}{l}40.6 \pm \\
1.3^{\mathrm{ab}}\end{array}$ \\
\hline & 10 & $\begin{array}{l}22.7 \pm \\
0.9^{\mathrm{a}}\end{array}$ & $\begin{array}{l}20.3 \pm \\
1.8^{\mathrm{ab}}\end{array}$ & $\begin{array}{l}19.0 \pm \\
1.7^{\mathrm{ab}}\end{array}$ & $\begin{array}{l}18.0 \pm \\
1.7^{\mathrm{ab}}\end{array}$ & $\begin{array}{l}16.1 \pm \\
1.0^{\mathrm{abc}}\end{array}$ & $\begin{array}{l}15.0 \pm \\
1.1^{b c}\end{array}$ & $\begin{array}{l}15.0 \pm \\
0.6^{\mathrm{cd}}\end{array}$ & $\begin{array}{l}13.3 \pm \\
0.3^{\mathrm{cd}}\end{array}$ & $\begin{array}{l}12.3 \pm \\
0.3^{c d}\end{array}$ & $\begin{array}{l}11.7 \pm \\
0.7^{\mathrm{d}}\end{array}$ & $\begin{array}{l}41.0 \pm \\
1.00^{\mathrm{ab}}\end{array}$ \\
\hline & 12 & $\begin{array}{l}22.4 \pm \\
0.9^{\mathrm{a}}\end{array}$ & $\begin{array}{l}20.0 \pm \\
0.6^{\mathrm{ab}}\end{array}$ & $\begin{array}{l}18.0 \pm \\
0.6^{\mathrm{ab}}\end{array}$ & $\begin{array}{l}17.0 \pm \\
0.6^{\mathrm{ab}}\end{array}$ & $\begin{array}{l}15.1 \pm \\
1.2^{\mathrm{abc}}\end{array}$ & $\begin{array}{l}14.3 \pm \\
1.4^{\mathrm{abc}}\end{array}$ & $\begin{array}{l}14.0 \pm \\
1.7^{\text {bcd }}\end{array}$ & $\begin{array}{l}12.7 \pm \\
1.2^{\text {bcd }}\end{array}$ & $\begin{array}{l}11.7 \pm \\
1.2^{\mathrm{cd}} \pm\end{array}$ & $\begin{array}{l}11.0 \pm \\
1.0^{\mathrm{d}}\end{array}$ & $\begin{array}{l}41.4 \pm \\
1.4^{\mathrm{ab}}\end{array}$ \\
\hline \multirow[t]{3}{*}{$\begin{array}{l}\text { Lecanicillium lecanii } \\
\text { MTCC } 956\end{array}$} & 8 & $\begin{array}{l}24.7 \pm \\
3.3^{a}\end{array}$ & $\begin{array}{l}20.3 \pm \\
2.6^{\mathrm{ab}}\end{array}$ & $\begin{array}{l}18.7 \pm \\
2.3^{\mathrm{ab}}\end{array}$ & $\begin{array}{l}17.8 \pm \\
2.3^{\mathrm{ab}}\end{array}$ & $\begin{array}{l}16.3 \pm \\
2.0^{b c}\end{array}$ & $\begin{array}{l}14.3 \pm \\
1.2^{\mathrm{abc}}\end{array}$ & $\begin{array}{l}13.0 \pm \\
0.6^{\mathrm{abcd}}\end{array}$ & $\begin{array}{l}11.3 \pm \\
0.3^{b c d}\end{array}$ & $\begin{array}{l}10.3 \pm \\
0.3^{\mathrm{bcd}}\end{array}$ & $\begin{array}{l}9.7 \pm \\
1.2^{\mathrm{bcd}}\end{array}$ & $\begin{array}{l}41.9 \pm \\
1.2^{\mathrm{ab}}\end{array}$ \\
\hline & 10 & $\begin{array}{l}23.3 \pm \\
0.9^{\mathrm{a}}\end{array}$ & $\begin{array}{l}19.0 \pm \\
0.6^{\mathrm{ab}}\end{array}$ & $\begin{array}{l}17.3 \pm \\
0.3^{\mathrm{ab}}\end{array}$ & $\begin{array}{l}16.3 \pm \\
0.3^{\mathrm{ab}}\end{array}$ & $\begin{array}{l}14.0 \pm \\
0.6^{\mathrm{abc}}\end{array}$ & $\begin{array}{l}12.3 \pm \\
0.3^{\mathrm{abc}}\end{array}$ & $\begin{array}{l}11.3 \pm \\
0.7^{\mathrm{abc}}\end{array}$ & $\begin{array}{l}10.0 \pm \\
0.6^{\mathrm{abc}}\end{array}$ & $\begin{array}{l}8.7 \pm \\
0.7^{\mathrm{abc}}\end{array}$ & $\begin{array}{l}8.5 \pm \\
0.9^{\mathrm{abcd}}\end{array}$ & $\begin{array}{l}42.2 \pm \\
1.7^{\mathrm{ab}}\end{array}$ \\
\hline & 12 & $\begin{array}{l}23.7 \pm \\
1.2^{\mathrm{a}}\end{array}$ & $\begin{array}{l}18.3 \pm \\
0.9^{\mathrm{ab}}\end{array}$ & $\begin{array}{l}17.0 \pm \\
0.6^{\mathrm{ab}}\end{array}$ & $\begin{array}{l}16.0 \pm \\
0.6^{\mathrm{ab}}\end{array}$ & $\begin{array}{l}13.3 \pm \\
1.2^{\mathrm{abc}}\end{array}$ & $\begin{array}{l}12.0 \pm \\
1.1^{\mathrm{abc}}\end{array}$ & $\begin{array}{l}11.1 \pm \\
1.1^{\mathrm{abc}}\end{array}$ & $\begin{array}{l}9.7 \pm \\
0.9^{\mathrm{abc}}\end{array}$ & $\begin{array}{l}8.7 \pm \\
0.9^{\mathrm{abc}}\end{array}$ & $\begin{array}{l}8.3 \pm \\
0.9^{\mathrm{abcd}}\end{array}$ & $\begin{array}{l}42.4 \pm \\
2.3^{\mathrm{ab}}\end{array}$ \\
\hline \multirow[t]{3}{*}{$\begin{array}{l}\text { Beauveria bassiana } \\
\text { Commercial formulation }\end{array}$} & 8 & $\begin{array}{l}23.3 \pm \\
1.8^{\mathrm{a}}\end{array}$ & $\begin{array}{l}20.7 \pm \\
0.7^{\mathrm{abc}}\end{array}$ & $\begin{array}{l}19.3 \pm \\
0.3^{\mathrm{ab}}\end{array}$ & $\begin{array}{l}19.0 \pm \\
0.6^{b}\end{array}$ & $\begin{array}{l}16.3 \pm \\
0.9^{b c}\end{array}$ & $\begin{array}{l}15.0 \pm \\
1.0^{b c}\end{array}$ & $\begin{array}{l}14.3 \pm \\
0.9^{b c d}\end{array}$ & $\begin{array}{l}12.7 \pm \\
0.9^{b c d}\end{array}$ & $\begin{array}{l}11.7 \pm \\
1.2^{\mathrm{cd}}\end{array}$ & $\begin{array}{l}11.0 \pm \\
1.1^{d}\end{array}$ & $\begin{array}{l}40.9 \pm \\
1.0^{\mathrm{ab}} \pm\end{array}$ \\
\hline & 10 & $\begin{array}{l}23.3 \pm \\
0.9^{\mathrm{a}}\end{array}$ & $\begin{array}{l}20.5 \pm \\
0.4^{\mathrm{abc}}\end{array}$ & $\begin{array}{l}19.1 \pm \\
0.5^{\mathrm{ab}}\end{array}$ & $\begin{array}{l}18.7 \pm \\
0.3^{\mathrm{ab}} \pm\end{array}$ & $\begin{array}{l}16.1 \pm \\
0.7^{\mathrm{abc}}\end{array}$ & $\begin{array}{l}14.7 \pm \\
1.2^{\mathrm{bc}} \pm\end{array}$ & $\begin{array}{l}14.0 \pm \\
1.5^{\text {bcd }}\end{array}$ & $\begin{array}{l}12.3 \pm \\
1.2^{b c d}\end{array}$ & $\begin{array}{l}11.3 \pm \pm \\
0.3^{b c d}\end{array}$ & $\begin{array}{l}10.7 \pm \\
1.2^{\text {cd }}\end{array}$ & $\begin{array}{l}40.7 \pm \\
1.0^{\mathrm{ab}}\end{array}$ \\
\hline & 12 & $\begin{array}{l}23.4 \pm \\
1.4^{\mathrm{a}}\end{array}$ & $\begin{array}{l}20.5 \pm \\
0.3^{\mathrm{abc}}\end{array}$ & $\begin{array}{l}18.9 \pm \\
0.5^{\mathrm{ab}}\end{array}$ & $\begin{array}{l}18.3 \pm \\
0.7^{\mathrm{ab}}\end{array}$ & $\begin{array}{l}15.7 \pm \\
0.3^{\mathrm{abc}}\end{array}$ & $\begin{array}{l}14.0 \pm \\
0.6^{\mathrm{abc}}\end{array}$ & $\begin{array}{l}13.3 \pm \\
1.2^{\mathrm{abcd}}\end{array}$ & $\begin{array}{l}11.7 \pm \\
1.2^{b c d}\end{array}$ & $\begin{array}{l}11.0 \pm \\
1.5^{\mathrm{bcd}}\end{array}$ & $\begin{array}{l}10.3 \pm \\
1.4^{\mathrm{bcd}}\end{array}$ & $\begin{array}{l}41.2 \pm \\
0.9^{\mathrm{ab}}\end{array}$ \\
\hline \multirow[t]{3}{*}{$\begin{array}{l}\text { Metarhizium anisopliae } \\
\text { Commercial formulation }\end{array}$} & 8 & $\begin{array}{l}24.7 \pm \\
5.2^{\mathrm{a}}\end{array}$ & $\begin{array}{l}22.0 \pm \\
3.6^{b c}\end{array}$ & $\begin{array}{l}20.7 \pm \\
2.9^{b}\end{array}$ & $\begin{array}{l}20.0 \pm \\
2.9^{b}\end{array}$ & $\begin{array}{l}17.3 \pm \\
1.8^{c}\end{array}$ & $\begin{array}{l}16.0 \pm \\
1.5^{c}\end{array}$ & $\begin{array}{l}14.9 \pm \\
1.5^{\mathrm{cd}}\end{array}$ & $\begin{array}{l}13.3 \pm \\
1.8^{\mathrm{cd}}\end{array}$ & $\begin{array}{l}13.0 \pm \\
2.1^{d}\end{array}$ & $\begin{array}{l}12.3 \pm \\
1.8^{\mathrm{d}}\end{array}$ & $\begin{array}{l}40.5 \pm \\
1.0^{\mathrm{ab}}\end{array}$ \\
\hline & 10 & $\begin{array}{l}21.4 \pm \\
0.9^{\mathrm{a}}\end{array}$ & $\begin{array}{l}19.0 \pm \\
0.6^{\mathrm{ab}}\end{array}$ & $\begin{array}{l}17.7 \pm \\
0.3^{\mathrm{ab}}\end{array}$ & $\begin{array}{l}17.0 \pm \\
0.6^{\mathrm{ab}}\end{array}$ & $\begin{array}{l}14.7 \pm \\
1.2^{\mathrm{abc}}\end{array}$ & $\begin{array}{l}13.7 \pm \\
0.7^{\mathrm{ab}}\end{array}$ & $\begin{array}{l}12.7 \pm \\
0.7^{\mathrm{abcd}}\end{array}$ & $\begin{array}{l}11.3 \pm \\
0.3^{b c d}\end{array}$ & $\begin{array}{l}11.0 \pm \\
1.5^{\mathrm{bcd}}\end{array}$ & $\begin{array}{l}10.3 \pm \\
1.8^{\mathrm{bcd}}\end{array}$ & $\begin{array}{l}40.6 \pm \\
1.6^{\mathrm{ab}}\end{array}$ \\
\hline & 12 & $\begin{array}{l}22.7 \pm \\
1.2^{a}\end{array}$ & $\begin{array}{l}20.0 \pm \\
0.6^{\mathrm{ab}}\end{array}$ & $\begin{array}{l}18.7 \pm \\
0.7^{\mathrm{ab}}\end{array}$ & $\begin{array}{l}17.9 \pm \\
0.6^{\mathrm{ab}}\end{array}$ & $\begin{array}{l}15.3 \pm \\
0.9^{\mathrm{abc}}\end{array}$ & $\begin{array}{l}14.0 \pm \\
0.6^{\mathrm{abc}}\end{array}$ & $\begin{array}{l}13.3 \pm \\
0.7^{\mathrm{abcd}}\end{array}$ & $\begin{array}{l}11.7 \pm \\
0.9^{b c d}\end{array}$ & $\begin{array}{l}11.3 \pm \\
0.9^{b c d}\end{array}$ & $\begin{array}{l}10.7 \pm \\
1.2^{\mathrm{cd}}\end{array}$ & $\begin{array}{l}41.2 \pm \\
1.5^{5 \mathrm{~b} \backslash}\end{array}$ \\
\hline \multirow[t]{3}{*}{$\begin{array}{l}\text { Lecanicillium lecanii } \\
\text { Commercial formulation }\end{array}$} & 8 & $\begin{array}{l}24.7 \pm \\
3.2^{\mathrm{a}}\end{array}$ & $\begin{array}{l}21.0 \pm \\
2.6^{\mathrm{abc}}\end{array}$ & $\begin{array}{l}19.0 \pm \\
2.6^{\mathrm{ab}}\end{array}$ & $\begin{array}{l}18.2 \pm \\
2.6^{\mathrm{ab}}\end{array}$ & $\begin{array}{l}16.7 \pm \\
2.3^{b c}\end{array}$ & $\begin{array}{l}14.7 \pm \\
1.4^{b c}\end{array}$ & $\begin{array}{l}13.3 \pm \\
0.9^{\mathrm{abcd}}\end{array}$ & $\begin{array}{l}11.7 \pm \\
0.7^{b c d}\end{array}$ & $\begin{array}{l}10.7 \pm \\
0.3^{b c d}\end{array}$ & $\begin{array}{l}10.0 \pm \\
1.5^{b c d}\end{array}$ & $\begin{array}{l}41.5 \pm \\
1.0^{\mathrm{ab}}\end{array}$ \\
\hline & 10 & $\begin{array}{l}23.3 \pm \\
0.9^{\mathrm{a}}\end{array}$ & $\begin{array}{l}19.7 \pm \\
0.7^{\mathrm{ab}}\end{array}$ & $\begin{array}{l}17.7 \pm \\
0.7^{\mathrm{ab}}\end{array}$ & $\begin{array}{l}16.7 \pm \\
0.3^{\mathrm{ab}}\end{array}$ & $\begin{array}{l}14.3 \pm \\
0.7^{\mathrm{abc}}\end{array}$ & $\begin{array}{l}12.7 \pm \\
0.3^{\mathrm{abc}}\end{array}$ & $\begin{array}{l}11.7 \pm \\
0.3^{\mathrm{abc}}\end{array}$ & $\begin{array}{l}10.3 \pm \\
0.3^{\mathrm{abcd}}\end{array}$ & $\begin{array}{l}9.7 \pm \\
0.9^{\mathrm{bcd}}\end{array}$ & $\begin{array}{l}9.2 \pm \\
1.5^{\mathrm{bcd}}\end{array}$ & $\begin{array}{l}42.1 \pm \\
0.7^{\mathrm{ab}}\end{array}$ \\
\hline & 12 & $\begin{array}{l}23.7 \pm \\
1.2^{\mathrm{a}}\end{array}$ & $\begin{array}{l}19.3 \pm \\
0.7^{\mathrm{ab}}\end{array}$ & $\begin{array}{l}17.3 \pm \\
0.7^{\mathrm{ab}}\end{array}$ & $\begin{array}{l}16.3 \pm \\
0.7^{\mathrm{ab}}\end{array}$ & $\begin{array}{l}14.0 \pm \\
0.6^{\mathrm{abc}}\end{array}$ & $\begin{array}{l}12.7 \pm \\
0.7^{\mathrm{abc}}\end{array}$ & $\begin{array}{l}11.4 \pm \\
0.8^{\mathrm{abc}}\end{array}$ & $\begin{array}{l}10.0 \pm \\
1.0^{\mathrm{abc}}\end{array}$ & $\begin{array}{l}9.3 \pm \\
0.3^{\mathrm{abc} c d}\end{array}$ & $\begin{array}{l}8.7 \pm \\
1.2^{\mathrm{bcd}}\end{array}$ & $\begin{array}{l}41.8 \pm \\
1.2^{2 \mathrm{~b}} \pm\end{array}$ \\
\hline \multirow[t]{2}{*}{$\begin{array}{l}\text { Azadirachtin 1\% (10,000 } \\
\text { ppm) }\end{array}$} & 4 & $\begin{array}{l}23.1 \pm \\
1.7^{\mathrm{a}}\end{array}$ & $\begin{array}{l}17.7 \pm \\
0.9^{\mathrm{ab}}\end{array}$ & $\begin{array}{l}16.3 \pm \\
0.9^{\mathrm{ab}}\end{array}$ & $\begin{array}{l}15.3 \pm \\
0.9^{\mathrm{ab}}\end{array}$ & $\begin{array}{l}12.7 \pm \\
0.3^{\mathrm{ab}}\end{array}$ & $\begin{array}{l}11.7 \pm \\
0.3^{\mathrm{ab}}\end{array}$ & $\begin{array}{l}11.0 \pm \\
0.6^{\mathrm{abc}}\end{array}$ & $\begin{array}{l}9.0 \pm \\
0.6^{\mathrm{ab}}\end{array}$ & $\begin{array}{l}7.7 \pm \\
0.3^{\mathrm{ab}}\end{array}$ & $\begin{array}{l}6.7 \pm \\
0.3^{\mathrm{abc}}\end{array}$ & $\begin{array}{l}44.0 \pm \\
1.7^{\mathrm{ab}}\end{array}$ \\
\hline & 5 & $\begin{array}{l}24.2 \pm \\
0.4^{a}\end{array}$ & $\begin{array}{l}17.7 \pm \\
1.3^{\mathrm{ab}}\end{array}$ & $\begin{array}{l}16.0 \pm \\
1.0^{\mathrm{a}}\end{array}$ & $\begin{array}{l}15.3 \pm \\
1.2^{\mathrm{ab}}\end{array}$ & $\begin{array}{l}13.0 \pm \\
0.6^{\mathrm{abc}}\end{array}$ & $\begin{array}{l}11.7 \pm \\
0.3^{\mathrm{ab}}\end{array}$ & $\begin{array}{l}10.7 \pm \\
0.3^{\mathrm{ab}}\end{array}$ & $\begin{array}{l}9.0 \pm \\
0.6^{\mathrm{ab}}\end{array}$ & $\begin{array}{l}7.7 \pm \\
0.3^{\mathrm{ab}}\end{array}$ & $\begin{array}{l}6.4 \pm \\
0.6^{\mathrm{ab}}\end{array}$ & $\begin{array}{l}44.4 \pm \\
4.4^{\mathrm{ab}}\end{array}$ \\
\hline $\begin{array}{l}\text { Chemical check } \\
\text { (malathion } 50 \mathrm{EC} \text { ) }\end{array}$ & 4 & $\begin{array}{l}23.3 \pm \\
1.85^{\mathrm{a}}\end{array}$ & $\begin{array}{l}16.7 \pm \\
1.7^{\mathrm{a}}\end{array}$ & $\begin{array}{l}15.0 \pm \\
1.0^{\mathrm{a}}\end{array}$ & $\begin{array}{l}14.2 \pm \\
0.9^{a}\end{array}$ & $\begin{array}{l}11.7 \pm \\
1.2^{\mathrm{a}}\end{array}$ & $\begin{array}{l}10.3 \pm \\
0.9^{\mathrm{a}}\end{array}$ & $9.7 \pm 0.9^{\mathrm{a}}$ & $7.1 \pm 0.5^{\mathrm{a}}$ & $\begin{array}{l}6.0 \pm \\
0.6^{\mathrm{a}}\end{array}$ & $\begin{array}{l}4.7 \pm \\
0.3^{\mathrm{a}}\end{array}$ & $\begin{array}{l}46.3 \pm \\
3.0^{\mathrm{a}}\end{array}$ \\
\hline Control & & $\begin{array}{l}24.0 \pm \\
2.1^{\mathrm{a}}\end{array}$ & $\begin{array}{l}25.3 \pm \\
1.3^{c}\end{array}$ & $\begin{array}{l}25.0 \pm \\
1.1^{c}\end{array}$ & $\begin{array}{l}25.3 \pm \\
1.4^{c}\end{array}$ & $\begin{array}{l}24.3 \pm \\
2.8^{\mathrm{d}}\end{array}$ & $\begin{array}{l}23.9 \pm \\
2.9^{\mathrm{d}}\end{array}$ & $\begin{array}{l}24.0 \pm \\
2.5^{e}\end{array}$ & $\begin{array}{l}24.7 \pm \\
2.1^{e}\end{array}$ & $\begin{array}{l}23.0 \pm \\
1.5^{e}\end{array}$ & $\begin{array}{l}22.0 \pm \\
0.6^{\mathrm{e}}\end{array}$ & $\begin{array}{l}40.0 \pm \\
1.0^{\mathrm{b}}\end{array}$ \\
\hline$F_{(18)}$ values & & 0.16 & 1.5 & 2.5 & 3.0 & 3.9 & 5.5 & 6.8 & 10.3 & 10.2 & 8.1 & 0.8 \\
\hline$P$ & & 1.00 & $>0.01$ & $<0.001$ & $<0.001$ & $<0.001$ & $<0.001$ & $<0.001$ & $<0.001$ & $<0.001$ & $<0.001$ & 0.7 \\
\hline
\end{tabular}

*Values represent means of 3 replicates

DAS days after spray

Means within each column bearing different letters are significantly different according to the Duncan test $(P=0.05)$

(2004) evaluated neem extract against the aphid $A$. gossypii pest on cotton and reported that aqueous extract of neem effected development, survival, and fecundity of A. gossypii thus causing high nymphal mortality.

\section{Biopesticide against $B$. tabaci}

The data presented in Table 3 showed that the whitefly population in the treatment control increased and decreased slightly with a maximum population density of 25.3 whiteflies per 3 leaves. After 10 days of 1st spray, 
Table 4 Percentage reduction of whitefly, Bemisia tabaci, on capsicum plant treated with indigenous and commercial bioformulations under protected cultivation $(2017,2018)$

\begin{tabular}{|c|c|c|c|c|c|c|c|c|c|c|}
\hline \multirow[t]{3}{*}{ Treatments } & \multirow{3}{*}{$\begin{array}{l}\text { Dose } \\
\text { (g or } \\
\mathrm{ml} / \mathrm{l})\end{array}$} & \multicolumn{9}{|c|}{ Percent reduction over control*(mean \pm SE) } \\
\hline & & \multicolumn{3}{|l|}{ I Spray } & \multicolumn{3}{|l|}{ II Spray } & \multicolumn{3}{|l|}{ III Spray } \\
\hline & & 3DAS & 7DAS & 10DAS & 3DAS & 7DAS & 10DAS & 3DAS & 7DAS & 10DAS \\
\hline \multirow[t]{3}{*}{$\begin{array}{l}\text { Beauveria bassiana } \\
\text { Bb-B1 }\end{array}$} & 8 & $\begin{array}{l}14.5 \pm \\
6.0^{\mathrm{a}}\end{array}$ & $\begin{array}{l}18.5 \pm \\
8.2^{c}\end{array}$ & $\begin{array}{l}22.3 \pm \\
8.3^{b}\end{array}$ & $26.3 \pm 3.1^{c}$ & $29.4 \pm 3.0^{d}$ & $31.2 \pm 5.4^{\mathrm{e}}$ & $40.2 \pm 7.9^{e}$ & $40.4 \pm 9.5^{e}$ & $\begin{array}{l}40.9 \pm \\
12.0^{\mathrm{d}}\end{array}$ \\
\hline & 10 & $\begin{array}{l}15.0 \pm \\
9.0^{\mathrm{a}}\end{array}$ & $\begin{array}{l}19.5 \pm \\
8.0^{b c}\end{array}$ & $\begin{array}{l}24.8 \pm \\
8.7^{\mathrm{ab}}\end{array}$ & $\begin{array}{l}30.0 \pm \\
10.7^{\mathrm{bc}}\end{array}$ & $\begin{array}{l}33.5 \pm \\
10.0^{c d}\end{array}$ & $33.8 \pm 7.9^{\text {de }}$ & $42.7 \pm 6.4^{\text {de }}$ & $43.2 \pm 4.9^{\text {de }}$ & $43.8 \pm 6.4^{\mathrm{C}}$ \\
\hline & 12 & $\begin{array}{l}15.6 \pm \\
5.4^{\mathrm{a}}\end{array}$ & $\begin{array}{l}23.0 \pm \\
7.3^{\mathrm{abc}}\end{array}$ & $\begin{array}{l}28.2 \pm \\
6.3^{\mathrm{ab}}\end{array}$ & $\begin{array}{l}33.6 \pm \\
9.4^{\mathrm{abc}}\end{array}$ & $\begin{array}{l}35.8 \pm \\
8.8^{\mathrm{bcd}}\end{array}$ & $\begin{array}{l}37.6 \pm \\
11.1^{\text {cde }}\end{array}$ & $\begin{array}{l}45.1 \pm \\
8.1^{\text {cde }}\end{array}$ & $45.8 \pm 8.5^{\text {de }}$ & $46.5 \pm 9.7^{\circ}$ \\
\hline \multirow[t]{3}{*}{$\begin{array}{l}\text { Lecanicillium lecanii } \\
\text { MTCC } 956\end{array}$} & 8 & $\begin{array}{l}21.9 \pm \\
5.2^{\mathrm{a}}\end{array}$ & $\begin{array}{l}27.3 \pm \\
3.8^{\mathrm{abc}}\end{array}$ & $\begin{array}{l}31.3 \pm \\
4.4^{\mathrm{ab}}\end{array}$ & $\begin{array}{l}34.7 \pm \\
5.4^{\mathrm{abc}}\end{array}$ & $\begin{array}{l}41.6 \pm \\
1.2^{\mathrm{abcd}}\end{array}$ & $\begin{array}{l}47.3 \pm \\
0.5^{\mathrm{abc} c \mathrm{e}}\end{array}$ & $\begin{array}{l}55.3 \pm \\
1.2^{\text {abcde }}\end{array}$ & $\begin{array}{l}56.3 \pm \\
4.0 \text { abcde }\end{array}$ & $\begin{array}{l}57.2 \pm \\
0.9^{\mathrm{abcd}}\end{array}$ \\
\hline & 10 & $\begin{array}{l}23.0 \pm \\
4.0^{\mathrm{a}}\end{array}$ & $\begin{array}{l}29.0 \pm \\
2.0^{\mathrm{abc}}\end{array}$ & $\begin{array}{l}33.7 \pm \\
2.6^{\mathrm{ab}}\end{array}$ & $\begin{array}{l}40.8 \pm \\
3.8^{\mathrm{abc}}\end{array}$ & $\begin{array}{l}46.8 \pm \\
1.8^{\mathrm{abc}}\end{array}$ & $51.4 \pm 0.6^{\mathrm{abc}}$ & $\begin{array}{l}58.3 \pm \\
1.5^{\mathrm{abcd}}\end{array}$ & $\begin{array}{l}61.2 \pm \\
3.2^{\mathrm{abcd}}\end{array}$ & $\begin{array}{l}60.0 \pm \\
4.8^{\mathrm{abcd}}\end{array}$ \\
\hline & 12 & $\begin{array}{l}26.6 \pm \\
7.4^{\mathrm{a}}\end{array}$ & $\begin{array}{l}31.0 \pm \\
6.4^{4 \mathrm{abc}}\end{array}$ & $\begin{array}{l}35.9 \pm \\
5.4^{\mathrm{ab}}\end{array}$ & $\begin{array}{l}44.4 \pm \\
2.7^{\mathrm{abc}}\end{array}$ & $\begin{array}{l}49.0 \pm \\
3.6^{\mathrm{ab}}\end{array}$ & $53.1 \pm 2.5^{\mathrm{abc}}$ & $\begin{array}{l}60.3 \pm \\
3.0^{\mathrm{abcd}}\end{array}$ & $\begin{array}{l}61.8 \pm \\
3.8^{\mathrm{abcd}}\end{array}$ & $\begin{array}{l}61.6 \pm \\
4.7^{\mathrm{abcd}}\end{array}$ \\
\hline \multirow[t]{3}{*}{$\begin{array}{l}\text { Beauveria bassiana } \\
\text { Commercial formulation }\end{array}$} & 8 & $\begin{array}{l}16.1 \pm \\
2.1^{\mathrm{a}}\end{array}$ & $\begin{array}{l}20.4 \pm \\
4.8^{\mathrm{abc}}\end{array}$ & $\begin{array}{l}22.8 \pm \\
5.8^{\mathrm{b}}\end{array}$ & $\begin{array}{l}30.9 \pm \\
2.5^{\text {bc }}\end{array}$ & $\begin{array}{l}35.4 \pm \\
2.6^{\text {bcd }}\end{array}$ & $\begin{array}{l}38.6 \pm \\
3.3^{\text {bcde }}\end{array}$ & $\begin{array}{l}47.2 \pm \\
4.0^{\text {bcde }}\end{array}$ & $\begin{array}{l}47.9 \pm \\
3.9^{\text {cde }}\end{array}$ & $\begin{array}{l}48.6 \pm \\
5.4^{\text {cd }}\end{array}$ \\
\hline & 10 & $\begin{array}{l}16.5 \pm \\
5.0^{\mathrm{a}}\end{array}$ & $\begin{array}{l}21.3 \pm \pm \\
3.4^{\mathrm{abc}}\end{array}$ & $\begin{array}{l}24.2 \pm \\
2.4^{\mathrm{ab}}\end{array}$ & $\begin{array}{l}31.8 \pm \\
2.6^{\mathrm{abc}}\end{array}$ & $\begin{array}{l}36.8 \pm \pm \\
3.6^{\text {bcd }}\end{array}$ & $\begin{array}{l}39.6 \pm \\
7.1^{\text {bcde }}\end{array}$ & $\begin{array}{l}48.6 \pm \pm \\
6.3^{\text {bcde }}\end{array}$ & $\begin{array}{l}49.3 \pm \\
2.7^{\text {bcde }}\end{array}$ & $\begin{array}{l}50.2 \pm \\
5.1^{b c d}\end{array}$ \\
\hline & 12 & $\begin{array}{l}16.9 \pm \\
8.6^{\mathrm{a}}\end{array}$ & $\begin{array}{l}22.7 \pm \\
7.2^{\mathrm{abc}}\end{array}$ & $\begin{array}{l}25.9 \pm \\
6.0^{\mathrm{ab}}\end{array}$ & $\begin{array}{l}34.1 \pm \\
11.5^{\mathrm{abc}}\end{array}$ & $\begin{array}{l}40.0 \pm \\
9.3^{\text {bcd }}\end{array}$ & $\begin{array}{l}43.1 \pm \\
11.8^{\mathrm{abcde}}\end{array}$ & $\begin{array}{l}51.6 \pm \\
12.7^{\mathrm{bcde}}\end{array}$ & $\begin{array}{l}51.1 \pm \\
12.8^{\mathrm{bcde}}\end{array}$ & $\begin{array}{l}52.0 \pm \\
11.8^{\mathrm{bcd}}\end{array}$ \\
\hline \multirow[t]{3}{*}{$\begin{array}{l}\text { Metarhizium anisopliae } \\
\text { Commercial formulation }\end{array}$} & 8 & $\begin{array}{l}15.5 \pm \\
3.5^{\mathrm{a}}\end{array}$ & $\begin{array}{l}19.6 \pm \\
6.5^{c}\end{array}$ & $\begin{array}{l}23.2 \pm \\
6.6^{b}\end{array}$ & $\begin{array}{l}30.7 \pm \\
2.7^{b c}\end{array}$ & $\begin{array}{l}34.8 \pm \\
3.6^{\mathrm{cd}}\end{array}$ & $\begin{array}{l}40.0 \pm \\
3.5^{\text {bcde }}\end{array}$ & $\begin{array}{l}47.4 \pm \\
0.7^{\text {bcde }}\end{array}$ & $45.0 \pm 1.9^{\text {de }}$ & $45.4 \pm 3.0^{\mathrm{d}}$ \\
\hline & 10 & $\begin{array}{l}16.0 \pm \\
2.1^{\mathrm{a}}\end{array}$ & $\begin{array}{l}21.0 \pm \\
0.5^{\mathrm{abc}}\end{array}$ & $\begin{array}{l}24.9 \pm \\
3.5^{\mathrm{ab}}\end{array}$ & $\begin{array}{l}32.5 \pm \\
2.6^{\mathrm{abc}}\end{array}$ & $\begin{array}{l}36.0 \pm \\
4.0^{\text {bcd }}\end{array}$ & $\begin{array}{l}40.9 \pm \\
3.8^{\text {abcde }}\end{array}$ & $\begin{array}{l}48.6 \pm \\
6.0^{\text {bcde }}\end{array}$ & $\begin{array}{l}46.5 \pm \\
10.3^{\mathrm{de}}\end{array}$ & $\begin{array}{l}47.4 \pm \\
11.0^{c d}\end{array}$ \\
\hline & 12 & $\begin{array}{l}16.4 \pm \\
3.3^{\mathrm{a}}\end{array}$ & $\begin{array}{l}21.0 \pm \\
4.2^{\mathrm{abc}}\end{array}$ & $\begin{array}{l}25.2 \pm \\
4.4^{\mathrm{ab}}\end{array}$ & $\begin{array}{l}33.3 \pm \\
6.9^{\mathrm{abc}}\end{array}$ & $\begin{array}{l}37.9 \pm \\
4.5^{\mathrm{bcd}}\end{array}$ & $\begin{array}{l}41.2 \pm \\
5.1 \mathrm{abcde}\end{array}$ & $\begin{array}{l}50.0 \pm \\
5.8^{\text {bcde }}\end{array}$ & $\begin{array}{l}47.8 \pm \\
4.7^{\text {cde }}\end{array}$ & $\begin{array}{l}48.7 \pm \\
4.6^{\mathrm{cd}} \pm\end{array}$ \\
\hline \multirow[t]{3}{*}{$\begin{array}{l}\text { Lecanicillium lecanii } \\
\text { Commercial formulation }\end{array}$} & 8 & $\begin{array}{l}19.3 \pm \\
4.4^{\mathrm{a}}\end{array}$ & $\begin{array}{l}26.0 \pm \\
4.2^{\mathrm{abc}}\end{array}$ & $\begin{array}{l}30.0 \pm \\
4.9^{\mathrm{ab}}\end{array}$ & $\begin{array}{l}33.4 \pm \\
6.7^{\mathrm{abc}}\end{array}$ & $\begin{array}{l}40.2 \pm \\
2.4^{\mathrm{abcd}}\end{array}$ & $\begin{array}{l}45.9 \pm \\
1.4^{\mathrm{abcde}}\end{array}$ & $\begin{array}{l}54.0 \pm \\
1.1^{\text {abcde }}\end{array}$ & $\begin{array}{l}54.8 \pm \\
2.8^{\mathrm{bcde}}\end{array}$ & $\begin{array}{l}55.8 \pm \\
2.0^{\mathrm{abcd}}\end{array}$ \\
\hline & 10 & $\begin{array}{l}20.1 \pm \\
3.8^{\mathrm{a}}\end{array}$ & $\begin{array}{l}27.3 \pm \\
2.6^{\mathrm{abc}}\end{array}$ & $\begin{array}{l}32.3 \pm \\
2.7^{\mathrm{ab}}\end{array}$ & $\begin{array}{l}39.4 \pm \\
5.3^{\mathrm{abc}}\end{array}$ & $\begin{array}{l}45.5 \pm \\
3.4^{\mathrm{abcd}}\end{array}$ & $\begin{array}{l}50.0 \pm \\
1.1^{\mathrm{abcd}}\end{array}$ & $\begin{array}{l}57.0 \pm \\
1.8^{\text {abcde }}\end{array}$ & $\begin{array}{l}56.7 \pm \\
4.5^{\text {abcde }}\end{array}$ & $\begin{array}{l}57.0 \pm \\
7.0^{\mathrm{abcd}}\end{array}$ \\
\hline & 12 & $\begin{array}{l}22.6 \pm \\
6.2^{\mathrm{a}}\end{array}$ & $\begin{array}{l}29.7 \pm \\
6.2^{\mathrm{abc}}\end{array}$ & $\begin{array}{l}34.6 \pm \\
5.4^{\mathrm{ab}}\end{array}$ & $\begin{array}{l}41.6 \pm \\
0.7^{\mathrm{abc}}\end{array}$ & $\begin{array}{l}46.2 \pm \\
2.4^{\mathrm{abc}}\end{array}$ & $51.6 \pm 1.5^{\mathrm{abc}}$ & $\begin{array}{l}59.0 \pm \\
4.1^{\mathrm{abcd}}\end{array}$ & $\begin{array}{l}58.9 \pm \\
0.9^{\text {abcde }}\end{array}$ & $\begin{array}{l}60.1 \pm \\
5.7^{\mathrm{abcd}}\end{array}$ \\
\hline \multirow[t]{2}{*}{$\begin{array}{l}\text { Azadirachtin 1\% (10,000 } \\
\text { ppm) }\end{array}$} & 4 & $\begin{array}{l}27.6 \pm \\
2.4^{\mathrm{a}}\end{array}$ & $\begin{array}{l}32.2 \pm \\
1.2^{\mathrm{abc}}\end{array}$ & $\begin{array}{l}37.1 \pm \\
1.8^{\mathrm{ab}}\end{array}$ & $\begin{array}{l}46.0 \pm \\
2.7^{\mathrm{abc}}\end{array}$ & $\begin{array}{l}49.3 \pm \\
2.4^{\mathrm{ab}}\end{array}$ & $52.4 \pm 1.4^{\mathrm{abc}}$ & $\begin{array}{l}62.1 \pm \\
2.8^{\mathrm{abc}}\end{array}$ & $\begin{array}{l}65.4 \pm \\
0.7^{\mathrm{abc}}\end{array}$ & $\begin{array}{l}68.5 \pm \\
2.0^{\mathrm{abc}}\end{array}$ \\
\hline & 5 & $\begin{array}{l}30.9 \pm \\
8.3^{\mathrm{a}}\end{array}$ & $\begin{array}{l}36.6 \pm \\
6.5^{\mathrm{ab}}\end{array}$ & $\begin{array}{l}40.0 \pm \\
7.0^{\mathrm{ab}}\end{array}$ & $\begin{array}{l}47.0 \pm \\
6.5^{\mathrm{ab}}\end{array}$ & $\begin{array}{l}51.6 \pm \\
4.1^{\mathrm{ab}}\end{array}$ & $56.0 \pm 4.2^{\mathrm{ab}}$ & $63.8 \pm 3.7^{\mathrm{ab}}$ & $67.1 \pm 1.1^{\mathrm{ab}}$ & $\begin{array}{l}71.0 \pm \\
1.6^{\mathrm{ab}}\end{array}$ \\
\hline $\begin{array}{l}\text { Chemical check (malathion } \\
50 \mathrm{EC} \text { ) }\end{array}$ & 4 & $\begin{array}{l}32.3 \pm \\
4.9^{\mathrm{a}}\end{array}$ & $\begin{array}{l}38.3 \pm \\
3.3^{\mathrm{a}}\end{array}$ & $\begin{array}{l}42.2 \pm \\
4.4^{\mathrm{a}}\end{array}$ & $50.7 \pm 3.6^{\mathrm{a}}$ & $55.5 \pm 3.1^{\mathrm{a}}$ & $58.6 \pm 2.1^{\mathrm{a}}$ & $70.4 \pm 2.2^{a}$ & $73.2 \pm 0.3^{\mathrm{a}}$ & $78.2 \pm 2.8^{\mathrm{a}}$ \\
\hline$F$ values & & 1.1 & 1.3 & 1.4 & 1.5 & 2.4 & 2.4 & 2.3 & 2.8 & 2.5 \\
\hline$P$ & & 0.40 & 0.22 & 0.20 & 0.14 & 0.01 & 0.01 & 0.01 & 0.01 & 0.01 \\
\hline
\end{tabular}

Mean mortality (\%) \pm standard error of $B$. tabaci recorded at different time intervals (DAS days after spray) for bioassays performed with different commercial and indigenous strains

Treatment columns bearing different alphabets are significantly different from other treatments according to Duncan test $(P=0.05)$

${ }^{*}$ Calculated as per Henderson and Tilton (1955) formula

whitefly population declined in all the mycoformulation treatments (16.0-18.7 whiteflies per 3 leaves) and in Azadirachtin 1\% (15.3 whiteflies per 3 leaves). The whitefly population infestation decreased gradually throughout the experiment. Both commercial and indigenous bioformulation decreased the whitefly population after 3rd spray. The whitefly population in L. lecanii
MTCC 956 at $12 \mathrm{~g} / \mathrm{l}$ was $9.7,8.7$, and 8.3 with $60.3,61.8$, and $61.6 \%$ population reduction after 3,7 , and 10 days after spray (DAS), respectively and was non-significant with MTCC 956 at $10 \mathrm{~g} / \mathrm{l}$ which recorded 10.0, 8.7, and 8.5 whiteflies per 3 leaves with 58.3, 61.2, and $60.0 \%$ population reduction after 3,7 , and 10 DAS, respectively (Table 4). Azadirachtin at 4 and $5 \mathrm{ml} / \mathrm{l}$ were non- 


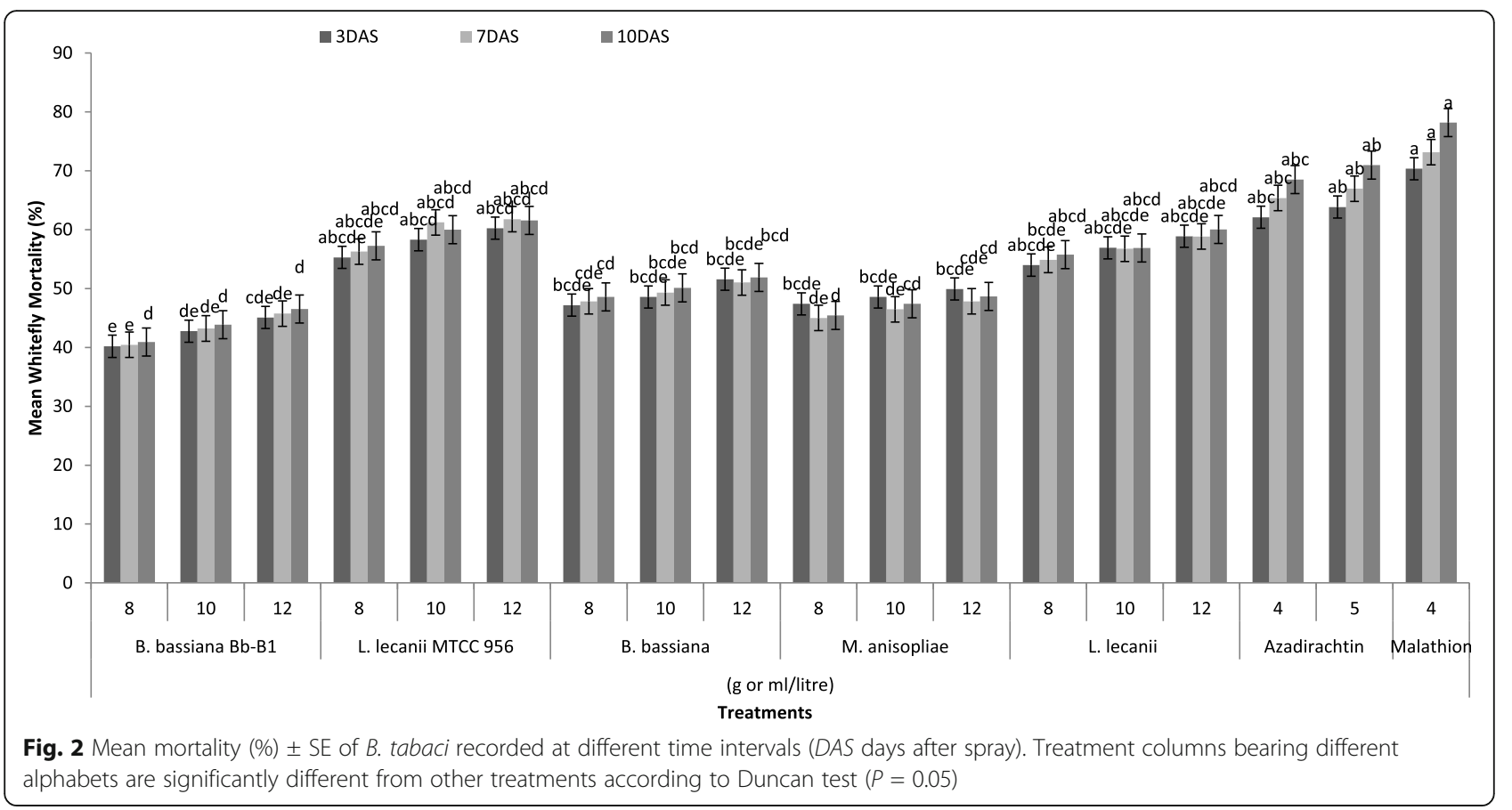

significant with each other and with L. lecanii MTCC 956 formulation. Azadirachtin $5 \mathrm{ml} / \mathrm{l}$ recorded whitefly population of 9.0, 7.7, and 6.4 whiteflies per 3 leaves per plant with $63.8,67.1$, and $71.0 \%$ whitefly population reduction after 3, 7, and 10 days after 3rd spray (Fig. 2). In the present study, it was observed that Azadirachtin 1\% at 4 and $5 \mathrm{ml} / \mathrm{l}$ and L. lecanii bioformulation at 10 and $12 \mathrm{~g} / \mathrm{l}$ was significantly better than all other fungal formulations in management of whitefly. So, we conclude that bioformulations of $L$. lecanii and azadirachtin $1 \%$ against $B$. tabaci resulted in a significant increase in the mortality of B. tabaci population under protected cultivation. The highest fruit yield was in all treatments were at par with each other. Other scientists' work (Cuthbertson and Walters, 2005) demonstrated that the pathogenicity of commercial EPF Lecanicillium muscarium (Mycotal, Koppert Biological Systems Ltd., UK) against sweet potato whitefly B. tabaci under laboratory and glasshouse cultivation and recorded that the application of $L$. muscarium against B. tabaci resulted in a significant increase in the mortality of $B$. tabaci under glasshouse cultivation and also no plant damage was recorded during the experiment. Budha et al. 2015 tested the efficacy of bio-pesticide against whitefly $B$. tabaci on tomato plant and they observed the efficacy test of some bio-pesticides on tomato plants to control nymphs of Tobacco whitefly $B$. tabaci, thus concluded that biopesticides viz. Bio Magic (91.64\%), Mealikil (93.55\%), and Biopower (88.91) were highly effective in killing nymphal whitefly population over control after 3rd spray. The most effective EPF that reduced the pest population were B. bassiana, $V$. lecanii, and $M$. anisopliae. These studies are in accordance with the present work where L. lecanii was recorded most effective against B. tabaci. Abdel-Rahim and Ahmed (2017) evaluated the EPF, $M$. anisopliae, $B$. bassiana, and $V$. lecanii at three different concentrations $\left(1 \times 10^{7}, 1 \times 10^{8}\right.$, and $1 \times 10^{9}$ spores $\left./ \mathrm{ml}\right)$ against $B$. tabaci in laboratory and field cultivation, respectively. They reported that higher concentration $(1 \times$ $10^{9}$ spores $/ \mathrm{ml}$ ) of the $3 \mathrm{EPF}$ was highly toxic to adults of $B$. tabaci than the other 2 concentrations. Under field cultivation also higher concentration $\left(1 \times 10^{9}\right)$ of $V$. lecanii was best in managing adult whitefly population; similar trend was found in this study also.

\section{Conclusion}

Obtained results showed that EPF, L. lecanii MTCC956 formulation was virulent to both $M$. persicae and $B$. tabaci on capsicum under protected cultivation when applied at 10 and $12 \mathrm{~g} / \mathrm{l}$. Similarly, botanical formulation Azadirachtin $1 \%$ at 4 and $5 \mathrm{ml} / \mathrm{l}$ was most effective in reducing $M$. persicae and B. tabaci on capsicum under protected cultivation. These results suggest that these biorationals can be included in integrated pest management programs designed for controlling the aphid and whitefly population on capsicum under protected conditions.

\section{Abbreviations}

PDA: Potato dextrose agar; RBD: Randomized block design; EC: Emulsifiable concentrate; N: Insect population; T: Treated; Co: Control; ANOVA: Analysis of 
variance; DMRT: Duncan multiple range test; cfu: Colony forming unit; DAS: Days after spray; MTCC: Microbial type culture collection and gene bank; LSD: Least significant difference

\section{Acknowledgements}

Authors are thankful to Head, Department of Entomology for providing facility to conduct present experiment and special thanks to Dr. P S Shera, Senior Entomologist, Department of Entomology, Punjab Agricultural University, Ludhiana, for his valuable suggestions during present study.

\section{Ethical approval and consent to participate}

Not applicable

\section{Authors' contributions}

H S carried out the experiments, recorded data, interpreted the results, and wrote the manuscript. $\mathrm{N} J$ designed and supervised the experiments, provided technical guidance, and edited manuscript. All authors read and approved the final manuscript during present study.

\section{Funding}

Not applicable

\section{Availability of data and materials}

Not applicable

\section{Consent for publication}

Not applicable

\section{Competing interests}

The authors declare that they have no competing interests.

\section{Author details}

'Department of Microbiology, Punjab Agricultural University, Ludhiana, Punjab, India. ${ }^{2}$ Department of Entomology, Punjab Agricultural University, Ludhiana 141004, Punjab, India.

Received: 20 February 2020 Accepted: 19 May 2020

Published online: 02 June 2020

\section{References}

Abdel-Rahim MA, Lamya Ahmed AK (2017) Virulence of three entomopathogenic fungi against whitefly, Bemisia tabaci (Gennadius) (Hemiptera: Aleyrodidae) in tomato crop. J Entomol 14(4):155-159

Ali S, Farooqi MA, Sajjad A, Ullah MI, Qureshi AK, Siddique B, Waheed W, Sarfraz M, Ali A (2018) Compatibility of entomopathogenic fungi and botanical extracts against the wheat aphid, Sitobion avenae (Fab.) (Hemiptera: Aphididae). Egypt J Biol Pest Control 28:97

Ali S, Ullah Ml, Arshad M, Iftikhar Y, Saqib M, Afzal M (2017) Effect of botanicals and synthetic insecticides on Pieris brassicae (L., 1758) (Lepidoptera: Pieridae). Turkish J Entomol 41(3):275-284

Budha PB, Sharma M, Pradhan SB (2015) Efficacy test of bio-pesticides against tobacco whitefly Bemisia tabaci (Gennadius, 1889) on tomato plants in Nepal. J Inst Sci Tech 20(2):11-17

Cuthbertson AGS, Walters KFA (2005) Pathogenicity of the entomopathogenic fungus, Lecanicillium muscarium, against the sweet potato whitefly Bemisia tabaci under laboratory and glasshouse conditions. Mycopathologia 160:315-319

Frantz JD, Gardner J, Hoffmann MP, Jahn MM (2004) Greenhouse screening of Capsicum accessions for resistance to green peach Aphid (Myzus persicae). Hort Sci 39(6):1332-1335

Gabarty A, Salem HM, Fouda MA, Abas AA, Ibrahim AA (2014) Pathogenicity induced by the entomopathogenic fungi Beauveria bassiana and Metarhizium anisopliae in Agrotis ipsilon (Hufn.). J Radiat Res Appl Sci 7:95-100

Gupta JK, Bhatnagar A, Agarwal VK, Mukherjee S, Sharma BK (2016) Population dynamics and extent of damage due to pest complex on capsicum (Capsicum annum L.) under shade net house. J progress agric 7(2):101-106

Henderson CF, Tilton EW (1955) Tests with acaricides against the brow wheat mite. J Econ Entomol 48:157-161

Kaur A, Joshi N (2014) Conidial production of Beauveria bassiana on agricultural products and effect of storage temperature on its formulation. Afr J Microbiol Res 8:3164-3170
Manfrino RG, Gutierrez AC, Steinkraus DC, Salta CE, Lastra CCL (2014) Prevalence of entomophthorelean fungi (Entomophthromycota) of aphids (Hemiptera: aphidae) on solanaceous crops in Argentina. J Invertebr Pathol 121:21-23

Mohammed AA, Kadhim JH, Kamaluddin ZNA (2018) Selection of highly virulent entomopathogenic fungal isolates to control the greenhouse aphid species in Iraq. Egypt J Biol Pest Control 28(71):1-7

Pappas ML, Migkou F, Broufas GD (2013) Incidence of resistance to neonicotinoid insecticides in greenhouse populations of the whitefly, Trialeurodes vaporariorum (Hemiptera: Aleyrodidae) from Greece. Appl Entomol Zool 48: 373-378

Pilkington LJ, Messelink G, van Lenteren JC, Mottee KL (2010) Protected biological control: biological pest management in the greenhouse industry. Biol Control 52:216-220

Santos TM, Costa NP, Torres AL, Junior ALB (2004) Effect of neem extract on the cotton aphid. Pesq agropec bras 39(11):1071-1076

Sayed SM, Ali EF, Al-Otaibi SS (2019) Efficacy of indigenous entomopathogenic fungus, Beauveria bassiana (Balsamo) Vuillemin, isolates against the rose aphid, Macrosiphum rosae L. (Hemiptera: Aphididae) in rose production. Egypt J Biol Pest Control 29(19):1-7

SPSS (2015) IBM SPSS Statistics for Windows (Version 23.0). IBM Corp, Armonk Chicago, IL

Ullah Ml, Altaf N, Afzal M, Arshad M, Mehmood N, Majeed S, Ali S, Abdullah A (2019) Effects of entomopathogenic fungi on the biology of Spodoptera litura (Lepidoptera: Noctuidae) and its reduviid predator, Rhynocoris marginatus (Heteroptera: Reduviidae). Inter J Insect Sci 11:1-7

Vu VH, Hong SI, Kim K (2007) selection of entomopathogenic fungi for aphid control. J Biosci Bioeng 104(6):498-505

\section{Publisher's Note}

Springer Nature remains neutral with regard to jurisdictional claims in published maps and institutional affiliations.

\section{Submit your manuscript to a SpringerOpen ${ }^{\circ}$ journal and benefit from:}

- Convenient online submission

- Rigorous peer review

- Open access: articles freely available online

High visibility within the field

- Retaining the copyright to your article

Submit your next manuscript at $\boldsymbol{\nabla}$ springeropen.com 